\title{
Improving incremental balance in the GSI 3DVAR analysis system
}

Authors:

Ronald M. Errico

Ronald.M.Errico@nasa.gov

Runhua Yang

Runhua.Yang-1@nasa.gov

Daryl T. Kleist

Daryl.Kleist@noaa.gov

David F. Parrish

John C. Derber

David.Parrish@noaa.gov

Russ Treadon

John.Derber@noaa.gov

Russ.Treadon@noaa.gov

Submitted to: Monthly Weather Review

\begin{abstract}
The Gridpoint Statistical Interpolation (GSI) analysis system is a unified global/regional 3DVAR analysis code that has been under development for several years at the National Centers for Environmental Prediction (NCEP)/Environmental Modeling Center. It has recently been implemented into operations at NCEP in both the global and North American data assimilation systems (GDAS and NDAS). An important aspect of this development has been improving the balance of the analysis produced by GSI. The improved balance between variables has been achieved through the inclusion of a Tangent Linear Normal Mode Constraint (TLNMC). The TLNMC method has proven to be very robust and effective. The TLNMC as part of the global GSI system has resulted in substantial improvement in data assimilation both at NCEP and at the NASA Global Modeling and Assimilation Office (GMAO).
\end{abstract}

\section{Popular Summary}

Atmospheric wind, temperature, pressure, and moisture content do not behave independently. Although some observations of the atmosphere are directly concerned with only one or two of those components, the implications of those observations on the other components cannot be neglected. Otherwise, the picture of the atmosphere so produced would be inconsistent with reality, leading to poor descriptions of climate and inaccurate weather forecasts. For this reason, much attention to specifying the relationships among the various atmospheric components is required in order to advance the science of meteorology and its many social applications. 


\begin{abstract}
Although the critical importance of these relationships has been known for more than 60 years, as greater accuracy as been required in their application, their revision has been required. In the latest version of the data analysis scheme used to produce input for subsequently analyze climate at NASA and produce weather forecasts at NOAA, a better description of these relationships is now being utilized. In particular, especially outside the tropical region, a more realistic specification of the relationship between pressure at the bottom of the atmosphere and vertically averaged winds is specified. The result is shown to produce more accurate weather forecasts as indicated by a variety of measures. While the small increase in accuracy would be unperceived by the public, it is one of the many small steps required in the never-ending pursuit of more accurate atmospheric analysis and weather forecasts.
\end{abstract}




\title{
Improving incremental balance in the GSI 3DVAR analysis system
}

\author{
Daryl T. Kleist ${ }^{1}$, David F. Parrish, John C. Derber, and Russ Treadon \\ Environmental Modeling Center \\ National Centers for Environmental Prediction, Camp Springs, MD \\ Ronald M. Errico ${ }^{2}$ and Runhua Yang ${ }^{3}$ \\ Global Modeling and Assimilation Office, \\ NASA Goddard Space Flight Center, Greenbelt, MD \\ ${ }^{1}$ Science Applications International Corporation \\ ${ }^{2}$ Goddard Earth Sciences and Technology Center \\ University of Maryland, Baltimore County \\ ${ }^{3}$ Science Systems and Applications Incorporated
}

Submitted to Monthly Weather Review

09 April 2008

Corresponding author address: Daryl T. Kleist, NOAA Science Center \#207, 5200 Auth Rd., Camp Springs, MD 20746-4304.

Email: daryl.kleist@noaa.gov 


\section{ABSTRACT}

The Gridpoint Statistical Interpolation (GSI) analysis system is a unified global/regional 3DVAR analysis code that has been under development for several years at the National Centers for Environmental Prediction (NCEP)/Environmental Modeling Center. It has recently been implemented into operations at NCEP in both the global and North American data assimilation systems (GDAS and NDAS). An important aspect of this development has been improving the balance of the analysis produced by GSI. The improved balance between variables has been achieved through the inclusion of a Tangent Linear Normal Mode Constraint (TLNMC). The TLNMC method has proven to be very robust and effective. The TLNMC as part of the global GSI system has resulted in substantial improvement in data assimilation both at NCEP and at the NASA Global Modeling and Assimilation Office (GMAO).

\section{Introduction}

The generation of large amplitude, high frequency oscillations can be problematic for numerical weather prediction (NWP) forecasts. Large imbalances between variables in the initial conditions can lead to the propagation of unrealistic inertial-gravity waves in an NWP forecast. These imbalances can exist through the process of unconstrained variational data assimilation, by upsetting the internal dynamic balance of the NWP model, even with an apparently suitable constraint modeled in the background error covariance matrix. Although present noise levels are much less than they were 20-30 years ago, they are still large enough to degrade the data assimilation process unless further mitigated. Various initialization methods have been developed to counteract this problem, including analysis post-processing procedures such as digital filtering (Huang and Lynch 1993) and nonlinear normal mode initialization (Machenhauer 1977, Baer and Tribbia 1977). Recently, however, it has been shown to be 
beneficial to improve the balance between analysis variables through methods internal to the actual assimilation procedure, such as through the inclusion of a weak constraint in the cost function (Wee and Kuo 2004, Liang et al. 2007).

Though it has been shown that initialization procedures are extremely beneficial to NWP, they suffer from the fact that they undo part of the work that the assimilation had completed to fit the observational data set (Williamson et al., 1981, Errico et al., 1993). Variational approaches have been used to reduce the damage done by initialization after the analysis by taking into account analysis uncertainty (Daley, 1978, Williamson and Daley, 1983, Fillion and Temperton, 1989). However, it is to be expected that the best result can be obtained by including an initialization-like operator internal to the analysis itself. This was demonstrated by Gauthier and Thépaut (2001), and Wee and Kuo (2004), through the inclusion of a penalty term in the analysis that measured the distance between digitally-filtered and unfiltered model states. Normal mode initialization operators have been used with some success as part of a penalty term in a study by Courtier and Talagrand (1990).

Initial testing of the gridpoint statistical interpolation (GSI, Wu et al. 2002), with the National Centers for Environmental Prediction (NCEP) global spectral model, showed that without additional constraints, the results were inferior to the operational system. The operational global data assimilation system (GDAS) at NCEP used then was the spectral statistical interpolation (SSI, Parrish and Derber 1992). The multivariate component of the unconstrained GSI is not as well balanced as its SSI counterpart, which has the advantage of being able to better approximate a balance equation by being formulated in spectral space. As such, it was decided to develop and test a procedure for the GSI to overcome this deficit, and to 
improve the balance of the initial conditions and subsequent quality of forecasts, such that GSI could replace the SSI as part of the operational GDAS.

In this paper, we describe the development of a procedure aimed at improving the balance of the GSI analysis increment. The motivation that led to the development of the Tangent Linear Normal Mode Constraint (TLNMC) will be described in section 2. A derivation and description of this analysis constraint then follows. Results from single observation tests, as well as single analysis and fully cycled experiments will be described in section 4 . Finally, a summary and discussion of future plans will be presented.

\section{Motivation}

\section{a. Weak Constraint}

Variational assimilation, such as 3DVAR in GSI, attempts to find the solution that best fits observations within a time window to a background (Derber 1989), by minimizing a cost function defined as

$$
J(\mathbf{x})=\frac{1}{2} \mathbf{x}^{\mathrm{T}} \mathbf{B}^{-1} \mathbf{x}+\frac{1}{2}(\mathbf{H} \mathbf{x}-\mathbf{y})^{\mathrm{T}} \mathbf{R}^{-1}(\mathbf{H} \mathbf{x}-\mathbf{y})+J_{\mathrm{c}},
$$

where $\mathbf{x}$ is the analysis increment, $\mathbf{B}$ the background error covariance, $\mathbf{y}$ the residual observation (difference between the observation $y_{o}$ and background value $H\left[\mathbf{x}_{\mathbf{b}}\right]$ ), $\mathbf{R}$ the observation error covariance, and $\mathbf{H}$ the observation operators. Note that an additional generic constraint, $J_{\mathrm{c}}$, has been included. This $J_{\mathrm{c}}$ penalty term can take many forms, but can be designed such that it measures the amount of noise or imbalance generated by the other two terms. Previous studies have incorporated digital filters (Gauthier and Thépaut, 2001, Wee and Kuo 2004) or nonlinear normal mode initialization (NLNMI) (Courtier and Talagrand, 1990) operators in the design of this term. 
A principle problem with various forms of a weak dynamic constraint $J_{\mathrm{c}}$ is that the minimization problem becomes highly ill-conditioned when the weights for $J_{\mathrm{c}}$ are increased to a level which produces reasonable balance. Additionally, prescribing the correct weighting of a covariance matrix (analogous to $\mathbf{B}$ for the background term) for the constraint term can be quite difficult. As a result of these difficulties, the fit of observations to the analysis can become unacceptably degraded compared to the result without $J_{\text {c }}$.

\section{b. Review of Normal Mode Initialization}

Before describing the idea of the tangent linear normal mode constraint (TLNMC) as an alternative to a weak-constraint $J_{\mathrm{c}}$, a brief review of normal mode initialization will be presented. The underlying principle of linear and nonlinear normal mode initialization is that an atmospheric state can be divided into "slow" and "fast" parts, using the normal modes of a shallow water approximation to the adiabatic equations of motion linearized about a state of rest. The slow modes correspond closely to traditional geostrophic motion and the fast modes to eastand west-ward propagating gravity waves. These slow and fast modes were originally used for linear normal mode initialization (LNMI) by Williamson and Dickinson (1972) to project the initial atmospheric state onto the gravity modes. These were then subtracted from the original state, supposedly to remove gravity wave oscillations from the forecast. It was later shown by Machenhauer (1977) that LNMI only provided a small reduction in gravity wave noise in the forecast.

Instead of zeroing the gravity mode component of the state, Machenhauer (1977) [and independently Baer and Tribbia (1977)] were the first to observe, from empirical and analytic methods, that a much more effective balance could be achieved by projecting the tendencies of the full nonlinear model to gravity modes, and then deriving a correction to the state which 
makes the gravity mode tendencies small. In the case of the Machenhauer scheme, a simple Picard iterative technique was used to obtain a solution. This is what makes the procedure nonlinear-thus nonlinear normal mode initialization (NLNMI). This original work was highly successful and resulted in great activity and many variations/applications of NLNMI during the 1980's. But this all came to an abrupt end with the advent of digital filtering (Huang and Lynch, 1993), which was much easier to apply with generally more robust results.

Before continuing with a description of the current application of NMI methodology, it is natural to ask why use a complex procedure when the much simpler digital filter is just as effective. There are two main reasons: 1) GSI is currently a 3DVAR system. To compute the incremental tendencies in GSI, a simple model following the formulation in Juang 2005 is used. This simple model is used only to estimate an incremental tendency for a single time level, and not to do time integration, which is necessary for application of the digital filter. 2) By projecting directly onto gravity modes, scale selection is possible-an important consideration, since we are primarily interested in removing high frequency small scale oscillations, and also some medium fast oscillations with large vertical and horizontal scale. Small scale advected Rossby motions can have high frequencies, but these are not changed by altering only gravity wave modes. So the scale selection is a complex mixture of small and large space scales and medium to high frequency in time. In contrast, the digital filter is strictly time selective, and the choice of time periods that are filtered is constrained by the integration period.

\section{Tangent Linear Normal Mode Constraint (TLNMC) \\ a. Description of TLNMC}


For those readers interested in details of normal mode initialization, Daley (1991) is an excellent resource, with extensive references. Here we introduce the following compact notation which is sufficient to communicate the principle behind TLNMC:

$\mathbf{x}_{\mathrm{u}}:$ unbalanced control analysis increment state vector of length $n$

$\mathbf{x}_{\mathrm{c}}$ : TLNMC balanced analysis increment state vector of length $n$

y: observation innovation vector of length $p$

T: $n$ by $n$ matrix representing the tangent linear tendency model mentioned in section 2 .

F: $\quad m$ by $n$ matrix which projects an $n$ dimensional state vector to $m$ gravity modes $(m<n)$

D: $n$ by $m$ Machenhauer-style correction matrix used to reduce gravity mode tendencies

C = I - DFT: $n$ by $n$ operator equivalent to a single iteration of Machenhauer-type algorithm.

In terms of the above notation, adding TLNMC yields the following modification of eq. (2.1):

$$
J\left(\mathbf{x}_{\mathrm{u}}\right)=\frac{1}{2} \mathbf{x}_{\mathrm{u}}^{\mathrm{T}} \mathbf{B}^{-1} \mathbf{x}_{\mathrm{u}}+\frac{1}{2}\left(\mathbf{H} \mathbf{x}_{\mathrm{c}}-\mathbf{y}\right)^{\mathrm{T}} \mathbf{R}^{-1}\left(\mathbf{H} \mathbf{x}_{\mathrm{c}}-\mathbf{y}\right)
$$

where $\mathbf{x}_{\mathbf{c}}=\mathbf{C x}_{\mathrm{u}}$ is the TLNMC balanced analysis increment and $J_{\mathrm{c}}$ has been dropped. ${ }^{1}$ Note that only the analysis increment is balanced via the TLNMC.

An alternative form for (3.1), expressed entirely in $\mathbf{x}_{\mathbf{c}}$ is

$$
J\left(\mathbf{x}_{\mathrm{c}}\right)=\frac{1}{2} \mathbf{x}_{\mathrm{c}}^{\mathrm{T}} \mathbf{C}^{-\mathrm{T}} \mathbf{B}^{-1} \mathbf{C}^{-1} \mathbf{x}_{\mathrm{c}}+\frac{1}{2}\left(\mathbf{H} \mathbf{x}_{\mathrm{c}}-\mathbf{y}\right)^{\mathrm{T}} \mathbf{R}^{-1}\left(\mathbf{H} \mathbf{x}_{\mathrm{c}}-\mathbf{y}\right),
$$

where it is assumed that $\mathbf{C}$ is formally invertible (like $\mathbf{B}$, the inverse of $\mathbf{C}$ is never required in the actual minimization algorithm used by GSI). Then the background error covariance for the TLNMC balanced increment $\mathbf{x}_{\mathrm{c}}$ is $\mathbf{C B C} \mathbf{C}^{\mathrm{T}}$. Thus, while $\mathbf{B}$ as currently defined in GSI is fixed independent of the background state, $\mathbf{C B C}^{\mathrm{T}}$ is a flow dependent covariance. The addition of a

\footnotetext{
${ }^{1}$ A similar incremental normal mode balance operator was developed and tested independently by Fillion et al. (2007).
} 
weak-constraint $J_{\mathrm{c}}$ also modifies the effective background error so it is also flow dependent. However, the TLNMC operator $\mathbf{C}$ restricts the unbalanced vector $\mathbf{x}_{\mathbf{u}}$ as a contraction to the observation term. With this contraction, it might be expected that the condition of (3.2) is actually improved over (2.1) with zero weight given to the $J_{\mathrm{c}}$ term (see appendix A for a more extensive discussion of these points).

It is possible to apply $\mathbf{C}$ more than once, which would make it truly like the Machenhauer NLNMI, except that the procedure is linear because of the definition of $\mathbf{C}$. However this adds significantly to the cost with no apparent benefit over applying $\mathbf{C}$ just once.

\section{b. A practical implementation of TLNMC in GSI}

The current version of GSI still uses a library of spherical harmonic transforms to convert the input background state from spectral coefficients to grid variables and the inverse for output of the analysis variables. It was decided that the easiest way to implement TLNMC was to follow precisely the implementation of implicit normal mode initialization for spectral models (Temperton, 1989). This version of NLNMI does not require explicit computation of the horizontal normal modes. Temperton's purpose was not to actually use this method for initialization of spectral models, but to compare implicit methods to standard NLNMI. Implicit NMI methods, which do not require explicitly defined normal modes, were originally developed for initialization of regional models (Bourke and McGregor, 1983, Briere, 1982), for which normal modes are hard to define. However the assumptions made are somewhat more restrictive compared to direct use of normal modes.

The only modification to Temperton's spectral scheme is to replace the full nonlinear model with a tangent linear system which is applied to the analysis increment in GSI. It was only necessary to check the algebra in the published paper and then implement it in the GSI 
code. Thus the components F and D of the TLNMC operator $\mathbf{C}$ were constructed directly from the prescriptions in Temperton.

Because this is a full 3D system, it is necessary to first perform a vertical mode decomposition. There has been a long standing controversy about the best way to do this, with the problem being that to do the vertical mode transform, the number of degrees of freedom in the vertical is reduced by one. It turns out that for small perturbations (true by definition for TLNMC) there is a unique method of defining the problem, originally proposed by Machenhauer in his extension of NLNMI to full 3D models (see appendix B).

Using vertical modes transforms the $3 \mathrm{D}$ problem into a series of $2 \mathrm{D}$ shallow water problems, each with an associated vertical structure function and gravity wave phase speed. The first five vertical modes for a typical case are shown in Figure 1, and the corresponding gravity wave phase speeds are presented in Table 1. These five structures are all approximately barotropic in the troposphere, as a consequence of the high vertical resolution and model top.

In the current formulation of TLNMC in GSI, there are several adjustable parameters. These are 1) NVMODES_KEEP, the number of vertical modes to include, 2) NTLNMC, the number of times to apply the TLNMC operator C, 3) PERIOD_MAX, the maximum period cutoff in hours for the gravity wave modes to include within each vertical mode system, and 4) PERIOD_WIDTH, also in hours, which specifies the width of a transition zone. This transition zone assures that the horizontal modes are not abruptly cut off, but smoothly vary from used to not used. Note that we do not really have normal modes, just spectral coefficients. A period is assigned to each spectral coefficient based on its two dimensional wave number and vertical mode phase speed. A mask is constructed that is a function of this period and goes smoothly from 1 to 0 as period increases from PERIOD_MAX - PERIOD_WIDTH to PERIOD_MAX + 
PERIOD_WITDH. The spectral coefficients of tendencies are first multiplied by this mask, before applying the operator $\mathbf{D}$. The current settings for operational implementation of TLNMC are NVMODES_KEEP $=8$, NTLNMC $=1$, PERIOD_MAX $=6$ hours, and PERIOD_WIDTH $=$ 1.5 hours. In the Temperton (1989) formulation, there are three approximations tested, of which system "B" was chosen for the TLNMC application. This scheme was chosen as it is closest to the explicit normal mode formulation.

\section{Experimental Results}

a. Single observation tests

The assimilation of a single observation is often used to assess the impact of the assignment of observation error and background error weights, as well as their covariance structures. Due to their simplicity and easy interpretation, such experiments can also be useful for diagnosing potential problems as well as in evaluating analysis changes. Fig. 2a shows the resultant $500 \mathrm{hPa}$ temperature analysis increment, for a single $500 \mathrm{hPa}$ temperature observation at $45^{\circ} \mathrm{N}$ and $90^{\circ} \mathrm{W}$ with a $1 \mathrm{~K}$ innovation and $1 \mathrm{~K}$ observation error assimilated with no dynamic constraint. The resultant $500 \mathrm{hPa}$ temperature increment run with the TLNMC turned on (Fig. 2b) is qualitatively and quantitatively similar to the control with no constraint (Fig. 2a). However, the temperature increment is forced to be less circular than the control, increasing the north-south temperature gradient (Fig. 2b). This is also evident when looking at the difference between the TLNMC and control run (Fig. 2c), where a tri-pole pattern is evident in the difference field. The magnitude of the impact of including the TLNMC appears to be small however, on the order of $5 \%$ or less.

For an analysis with no constraint, the assimilation of a single temperature observation results in a wind increment consistent with the definition of the multivariate background error. 
In the case of the global GSI, the relationship between temperature and wind comes from statistically derived parameters using the so-called NMC method (Wu et al., 2002). A vertical cross section taken at $90^{\circ} \mathrm{W}$ (Fig. 3a) shows the zonal wind increment determined by the single temperature observation experiment. Through the variable definition and corresponding background error structure functions, a warm temperature observation has resulted in an anticyclonic circulation, with a maximum found near $300 \mathrm{hPa}$, above the location of the original temperature observation. The same experiment run with the TLNMC turned on increases this anti-cyclonic circulation substantially (Fig. 3b). The difference between the zonal wind increment for the TLNMC and control runs is as large as $35 \%$ at $300 \mathrm{hPa}$ (Fig. 3c).

The fact that the TLNMC increases the induced circulation from a single temperature observation suggests that perhaps the statistically derived background error structure functions are deficient or lacking proper flow-dependence. : For the mid-tropospheric mid-latitudes, it. would be expected that a mass-wind relationship for an analysis increment could be well described as nearly geostrophic, consistent with a thermal wind balance. Though the background error definitions seem to do a reasonable job in capturing a majority of this relationship, the TLNMC appears to clean up the deficiencies in the multivariate background error definition. By first converting a temperature increment to geopotential height increment, one can derive an ageostrophic wind increment by subtracting off the implied geostrophic component from the actual wind increment. For the case without the TLNMC, the single temperature observation resulted in a fairly strong ageostrophic zonal wind increment, particularly above the height of the observation (Fig. 4a). However, the TLNMC seems to be doing what it was designed to do in improving the balance between variables, by substantially reducing the ageostrophic component of the increment (Figs. $4 b$ and c). 


\section{b. Single analysis diagnosis}

Further evaluation of the impact of running an analysis using the TLNMC is done by performing a single analysis, utilizing the full suite of observational data, but starting from the same background field. A single analysis was performed for the 12 UTC GDAS cycle on 09 October 2007, assimilating all operational observations, including conventional and satellite brightness temperature data. The background used for the control and TLNMC assimilation experiments was the operational six hour forecast from the previous 06 UTC GDAS cycle. Similar to the single observation experiment, resultant analysis increments for a control with no constraint and an analysis that utilizes the TLNMC are qualitatively and quantitatively similar (Fig. 5).

The differences that result from using the TLNMC on a single analysis can be quite large however. For the $500 \mathrm{hPa}$ zonal wind increment for this case, the differences are sometimes upward of $50 \%$ of the increment (Fig. 6a). Though not shown, this is consistent with other variables and at other levels. Often times, the largest impact of the TLNMC can be found in the extratropics, and in particular, in regions of strong background flow, or regions of strong horizontal gradients (Fig 6). This has important implications, in that the use of such a constraint has in itself the ability to impose some notion of flow dependence. This has also been demonstrated by performing a series of single observation experiments, where the single observation is moved into different parts of the atmosphere where the background has differing characteristics (not shown).

Despite the differences resulting from the TLNMC appearing to be quite large, it is worth noting that the synoptic scale information content derived from the observations remains intact. This is demonstrated by comparing the mid-tropospheric meridional wind tendencies in the 
analyses with and without the TLNMC. The differences in these tendencies are small in magnitude and no discernable systematic pattern is evident (Fig. 7). The largest changes to the analysis increment seem to occur in the correction of the barotropic component, which allows for the analysis to fit the observations without removing their synoptic scale signature, while improving the incremental balance and removing noise.

The TLNMC was designed to remove noise from the analysis while improving the masswind relationship where appropriate. One symptom of some of the problems when running the GSI analysis with no constraints is a substantial increase in the amplitude of the zonal mean surface pressure tendency over that of the background (Fig. 8). However, by running the same analysis but with the TLNMC and utilizing the same background as the control assimilation, the problem essentially disappears. The analyzed zonal mean surface pressure tendency from the TLNMC is nearly identical to that derived from the background. This result is consistent with the reduction in the implied (derived) magnitude of the zonal mean RMS of the vertical velocity increment (Fig. 9). This suggests that the analysis from the TLNMC is therefore much more in sync with the model dynamics and how the atmosphere behaves, and much less likely to suffer from spin-up or spin-down.

One of the more exciting elements of the TLNMC is that the improved balance and noise reduction in the analysis increment is achieved without having much of an impact on the minimization itself. The reduction of the norm of the gradient of the cost function for the control analysis without constraint and the TLNMC analysis is nearly identical, with a final reduction of six orders of magnitude (Fig. 10a). However, this does not all happen without cost, as there does appear to be some impact on the reduction of the cost function (Fig. 10b). The impact on the final reduction of the cost function for this case is approximately $2 \%$. However, this small 
difference appears to be negligible in the shadows of the positive impact seen from the noise reduction and improved balance, and is in fact, much smaller than the impact on convergence seen in some weak constraint experiments.

\section{c. Cycled experiments}

Although the single analysis experiments seem to suggest that the quality of analyses is improved through the use of the TLNMC, it is important to evaluate the impact of including such a change on subsequent forecasts. To evaluate the impact of the new constraint on forecast skill, two experiments were run; one without constraint (CTL), and the other with TLNMC turned on (TLNMC). The experiments were designed to mimic NCEP operations, using the operational global forecast model (GFS) and complete suite of observations as well as the operational data pre-processing codes (as of 01 May 2007). The spectral forecast model was run at T382 truncation with 64 hybrid (sigma-pressure) vertical levels, and utilized a digital filter centered about 3 hours. The diabatic digital filter was part of all forecast model time integrations, including the shorter 9-hr forecasts used for the assimilation as well as the 8-day free forecasts, and was applied in both the CTL and TLNMC experiments. This means that the background for all analyses in both experiments came from model forecasts that had been altered by the digital filter. The GSI analyses were performed on the Gaussian-linear grid that corresponds to the T382 spectral truncation ( $768 \times 384$ horizontal grid points), and on the model vertical levels, using the operational background error statistics.

Each experiment was fully cycled, creating four analyses and subsequent 9-hr forecasts per day respectively. The forecast used as the background for each analysis comes from its own previous cycle. Since the quality control was performed independently for each experiment, more data can get into the system as the experiment moves forward in time, if the quality of 
forecasts can somehow be improved. Lastly, unlike in operations, the full medium range forecast initialized from the early data cut-off time was only run at 00 UTC, and not four times per simulated day. The experiments were run for 2 months, each starting from the operational GDAS analysis at 00 UTC 15 November 2006, but the first 2 weeks of results were disregarded for spin-up.

It is evident that the use of the TLNMC leads to an improvement of the quality of the short-term forecasts being used as the background for subsequent analysis. The TLNMC experiment shows a consistent reduction in the fit of surface pressure observations to the background, a six hour forecast, relative to the CTL (Fig. 11). However, the fit of these very same observations to the analysis seems to be slightly degraded relative to the no-constraint CTL experiment... This slight degradation is to be expected, as the TLNMC forces the analysis to maintain a sufficiently balanced analysis increment, and therefore, not allowing the analysis to draw as closely to the individual observations.

The biggest impact on forecast skill, as expected, can be found in the extratropics. In terms of $500 \mathrm{hPa}$ anomaly correlation, the TLNMC is an improvement over the CTL in both hemispheres (Fig. 12). In fact, in the southern hemisphere, the use of the TLNMC has increased the forecast skill by as much as one quarter to one half day in the medium range. The fact that the gain in the Southern Hemisphere is larger than that seen in the Northern Hemisphere may be seasonal and case dependent. On a forecast by forecast basis, the 5-day $500 \mathrm{hPa}$ anomaly correlation scores from the TLNMC run rarely has drop-outs as large as the control, and often significantly outperforms the CTL many days in a row (Fig. 13). In addition to the anomaly correlation, a consistent reduction in the geopotential height root mean square error is found (not 
shown). Lastly, results are similar when verification is computed for other levels and variables (not shown).

An improvement in the 3-day precipitation forecasts over North America was also found for the experiment that utilized the TLNMC (Fig. 14). The improvement in skill is small, but consistent, especially for low threshold amounts. However, some degradation in skill is noted for some of the higher threshold amounts. The precipitation verification for Day-1 and Day-2 is quite similar, though the differences are even smaller. It is noteworthy that for Day-3, there exists a substantial high bias for the highest threshold amounts (Fig. 14c). However, there are very few cases for this Northern Hemisphere winter period for this to be considered a meaningful result. The TLNMC has been run for other periods (Northern Hemisphere summer for example), and the high bias for high precipitation threshold amounts does not appear to be an issue.

The analysis differences for the single analysis experiments were quite small in the tropics, when comparing a no-constraint analysis with one that had the TLNMC turned on (Fig 6b). As such, it is not surprising that the forecast skill in the tropics is very similar between the TLNMC and CTL runs. There appears to be a small increase in the tropical wind vector RMS error at both $200 \mathrm{hPa}$ (Fig. 15a) and $850 \mathrm{hPa}$ (Fig. 15b). However, this increase is on average only about $1.5 \%$ for both pressure levels for a 3-day lead time for this forecast period.

\section{Summary and discussion}

Through the inclusion of a constraint based upon the ideas from normal mode initialization, the quality of analyses produced by the global GSI system has been greatly improved. Incremental noise reduction and balance improvement are achieved without having much of an impact at all on the minimization performed as part of the variational assimilation algorithm. This is a major advantage over analogous weak-constraint formulations, which are 
often ill-conditioned as the weights need to be increased substantially to achieve a reasonably balanced increment. Though other studies have shown some success through the inclusion of a digital filter-based weak constraint in the context of 4DVAR (Gauthier and Thepaut 2000, Wee and Kuo 2003), this is not possible to include in the context of 3DVAR, where no model time integration exists. However, the TLNMC is generic enough that it could be included as part of a 4DVAR scheme and be tested against a digital filter based weak-constraint.

The TLNMC was evaluated for single observation and individual analysis cases. It was shown that for such tests, the TLNMC improved the incremental balance relationship between mass and wind relative to analyses run with no constraints at all. Although the variable definition and multivariate background error correlations impose an approximate form of balance close to that of a linear balance relationship, through statistically derived parameters, the addition of the TLNMC makes corrections that yield a more realistic incremental balance between variables. In fact, initial tests were done to attempt to remove the multivariate component of the background error altogether. Though it appeared that the TLNMC captured a good portion of the balancing correctly, the results were not quite as good when compared with control TLNMC analyses which used the multivariate background parameters. However, it may still be possible to achieve this through the inclusion of more vertical modes or further tuning of other parameters. This will likely be an area of future work, as removing the multivariate component would greatly simplify the estimation and application of the background error covariances.

Once it was demonstrated that the TLNMC resulted in reduced noise as well as a more balanced analysis increment, a two month fully-cycled experiment was conducted to assess the impact on forecast skill. Utilizing the constraint had an immediate impact on the quality of both the short-term forecasts that are used as the background in subsequent analyses as well as the 
medium range forecasts. Consistently, the TLNMC run showed a reduction in the RMS of the observation fit to the background, particularly for surface pressure data. Additionally, a significant improvement was found in terms of extra-tropical anomaly correlation scores, particularly in the Southern Hemisphere for the period evaluated. However, other tests over other periods seem to show that the amount of improvement is case dependent. The impact of using the TLNMC on the tropical circulation seems minimal, though it is worth noting that there was a slight increase in the wind vector RMS error when compared against its own analyses.

The improvements gained through the inclusion of the TLNMC allowed for the operational implementation of the global GSI as part of the GDAS at NCEP on 01 May 2007, replacing the old SSI-based 3DVAR system. The TLNMC was also tested at the NASA Goddard Global Modeling and Assimilation Office, as part of GEOS-5 Data Assimilation System, where very similar improvements were found. Lastly, work is under way to include the TLNMC, or a very similar version, for the regional applications of GSI. 


\section{APPENDIX A \\ Background error for TLNMC and weak constraint}

It can be shown that the effective background error for a 3DVAR analysis that uses a TLNMC or weak constraint contains implicit flow-dependence. However, a further expansion shows that the use of a weak constraint instead of the TLNMC can complicate preconditioning and therefore lead to convergence problems.

The cost function for generic linear 3DVAR equations involving increments was shown in (3.1). The solution $\mathbf{x}_{\mathrm{u}}$ that minimizes $J$ is

$$
\mathbf{x}_{\mathrm{u}}=\mathbf{A} \mathbf{H}^{\mathrm{T}} \mathbf{R}^{-1} \mathbf{y}(\mathrm{A} 1)
$$

where

$$
\mathbf{A}=\left(\mathbf{B}^{-1}+\mathbf{H}^{\mathrm{T}} \mathbf{R}^{-1} \mathbf{H}\right)^{-1}
$$

is the analysis error covariance. The solution (A1) can be represented in the equivalent observation space form

$$
\mathbf{x}_{\mathrm{u}}=\mathbf{B H}^{\mathrm{T}}\left(\mathbf{H B H ^ { \mathrm { T } }}+\mathbf{R}\right)^{-1} \mathbf{y} \cdot(\mathrm{A} 3)
$$

In GSI, the actual variable solved for is $\mathbf{z}_{\mathrm{u}}$, where

$$
\mathbf{x}_{\mathrm{u}}=\mathbf{B z}_{\mathrm{u}} \cdot(\mathrm{A} 4)
$$

The solution in terms of $\mathbf{z}_{\mathbf{u}}$ is then defined as

$$
\mathbf{z}_{\mathrm{u}}=\mathbf{B}^{-1} \mathbf{A} \mathbf{H}^{\mathrm{T}} \mathbf{R}^{-1} \mathbf{y}(\mathrm{A} 5)
$$

where

$$
\mathbf{B}^{-1} \mathbf{A}=\left(\mathbf{I}+\mathbf{B H}^{\mathrm{T}} \mathbf{R}^{-1} \mathbf{H}\right)^{-1} \text {. (A6) }
$$


The GSI uses a conjugate gradient method to obtain the solution $\mathbf{z}_{\mathrm{u}}$. The convergence rate depends on the ratio of the smallest to largest eigenvalues of $\mathbf{B}^{-1} \mathbf{A}$, which is considerably smaller than that for $\mathbf{A}$.

For TLNMC, we use the definition $\mathbf{x}_{c}=\mathbf{C} \mathbf{x}_{u}$ from section $3 \mathrm{a}$, where $\mathbf{C}$ is the TLNMC balance operator. Solving for equation (3.2), we find that

$$
\mathbf{X}_{\mathrm{c}}=\mathbf{A}_{\mathrm{c}} \mathbf{H}^{\mathrm{T}} \mathbf{R}^{-1} \mathbf{y}
$$

where

$$
\mathbf{A}_{\mathrm{c}}=\left(\mathbf{C}^{-\mathrm{T}} \mathbf{B}^{-1} \mathbf{C}^{-1}+\mathbf{H}^{\mathrm{T}} \mathbf{R}^{-1} \mathbf{H}\right)^{-1}(\mathrm{~A} 8)
$$

The control variable used in this case to improve convergence is $\mathbf{z}_{\mathrm{c}}$, such that

$$
\mathbf{x}_{\mathrm{c}}=\mathrm{CBC}^{\mathrm{T}} \mathbf{z}_{\mathrm{c}} \text { (A9) }
$$

and

$$
\mathbf{z}_{\mathrm{c}}=\mathbf{C}^{-\mathrm{T}} \mathbf{B}^{-1} \mathbf{C}^{-1} \mathbf{A}_{\mathrm{c}} \mathbf{H}^{\mathrm{T}} \mathbf{R}^{-1} \mathbf{y}
$$

The convergence rate then depends on the ratio of eigenvalues of

$$
\mathbf{C}^{-\mathrm{T}} \mathbf{B}^{-1} \mathbf{C}^{-1} \mathbf{A}_{\mathbf{c}}=\left(\mathbf{I}+\mathbf{C B C} \mathbf{C}^{\mathrm{T}} \mathbf{H}^{\mathrm{T}} \mathbf{R}^{-1} \mathbf{H}\right)^{-1} \text {. (A11) }
$$

It should be noted that the TLNMC balance operator $\mathbf{C}$ reduces (or contracts) the number of degrees of freedom of the vector it operates on. Although $\mathbf{C}^{-1}$ is not really defined, in practice within the GSI algorithm only $\mathbf{C}$ needs to be applied. The observation space form of (A9) is

$$
\mathbf{x}_{\mathbf{c}}=\mathbf{C B C}^{\mathrm{T}} \mathbf{H}^{\mathrm{T}}\left(\mathbf{H C B C}^{\mathrm{T}} \mathbf{H}^{\mathrm{T}}+\mathbf{R}\right)^{-1} \mathbf{y} .(\mathrm{A} 12)
$$

For the solution $\mathbf{x}_{\mathrm{c}}$, the effective background error is then

$$
\mathrm{B}_{\mathrm{c}}=\mathrm{CBC}^{\mathrm{T}} \text {. (A13) }
$$

In GSI with TLNMC, we precondition by $\mathbf{B}_{\mathrm{c}}$ which has a smaller effective dimension than $\mathbf{B}$, so the convergence rate to $\mathbf{x}_{\mathrm{c}}$ should be no worse than that for $\mathbf{x}_{\mathrm{u}}$ (and possibly better). 
One may ask why the existing multivariate balance included as part of $\mathbf{B}$ does not also reduce the effective degrees of freedom from $\mathbf{B}$ without multivariate balance. The reason is that an unbalanced part is also defined as part of $\mathbf{B}$. However, with or without this unbalanced part, when $\mathbf{C}$ is applied, a new flow-dependent balance is strictly enforced, such that there is no unbalanced part. This is why the effective dimension is reduced with TLNMC and explains why it is not possible to fit observations as closely as is the case without TLNMC.

One can also look at a weak noise constraint in this manner, where the cost function would be defined as

$$
J_{w}(\mathbf{x})=\mathbf{x}^{\mathrm{T}} \mathbf{B}^{-1} \mathbf{x}+(\mathbf{H} \mathbf{x}-\mathbf{y})^{\mathrm{T}} \mathbf{R}^{-1}(\mathbf{H} \mathbf{x}-\mathbf{y})+(\mathbf{G} \mathbf{x})^{\mathrm{T}} \mathbf{W}^{-1}(\mathbf{G x}) .(\mathrm{A} 14)
$$

In equation (A14), $\mathbf{G}=\mathbf{F T}$ converts a state vector $\mathbf{x}$ into gravity mode tendencies. $\mathbf{W}$ is a weighting matrix, which as mentioned in section 2 , is difficult to determine in practice. This second term constitutes what would seem to be the ideal weak constraint term. The solution which minimizes $J_{\mathrm{w}}$ would be

$$
\mathbf{x}_{\mathrm{w}}=\mathbf{A}_{\mathbf{w}} \mathbf{H}^{\mathrm{T}} \mathbf{R}^{-1} \mathbf{y}
$$

where

$$
\mathbf{A}_{w}=\left(\mathbf{B}^{-1}+\mathbf{G}^{\mathrm{T}} \mathbf{W}^{-1} \mathbf{G}+\mathbf{H}^{\mathrm{T}} \mathbf{R}^{-1} \mathbf{H}\right)^{-1} .
$$

Notice that in this case, the effective background error covariance is now the inverse of a sum,

$$
\mathbf{B}_{\mathrm{w}}=\left(\mathbf{B}^{-1}+\mathbf{G}^{\mathrm{T}} \mathbf{W}^{-1} \mathbf{G}\right)^{-1} \text {. (A17) }
$$

It is no longer possible to use the simple preconditioning strategy which involves multiplication by the effective background error, and therefore convergence problems result for reasonable values of the weighting matrix $\mathbf{W}$, when $\mathbf{B}$ is used as the preconditioner. The observation space solution is identical to (A4) with $\mathbf{B}$ replaced by $\mathbf{B}_{\mathrm{w}}$. 


\section{APPENDIX B}

\section{Vertical mode decomposition}

To apply normal mode initialization to a 3D hydrostatic model, it is first necessary to define vertical eigenmodes which then transform the 3D system into a series of 2D shallow water models, whose depths are functions of the eigenvalues of the vertical modes. The problem that arises is that, for a sigma coordinate model, there are $\mathrm{N}+1$ apparent degrees of freedom defining the mass $-\mathrm{N}$ levels of temperature $\mathrm{T}$ as well as surface pressure $\mathrm{p}$. But only $\mathrm{N}$ vertical modes can be defined, leaving an apparent ambiguity defining $\mathrm{T}$ and $\mathrm{p}$ in terms of vertical modes. Machenhauer demonstrated an unambiguous way to recover the extra degree of freedom, which is valid for small perturbations. But since the linearization is only valid for small perturbations anyway, there really is no extra degree of freedom in the limit of infinitesimal perturbations.

Consider the following highly simplified system which contains the essence of the problem:

$$
\begin{aligned}
& \frac{\partial \delta}{\partial t}=-\nabla^{2} \varphi \quad(\mathrm{B} 1) \\
& \varphi=\mathbf{H p}+\mathrm{AT} \quad \text { (B2) } \\
& \frac{\partial \mathrm{T}}{\partial t}=-\mathbf{B} \delta \quad \text { (B3) } \\
& \frac{\partial \mathrm{p}}{\partial t}=-\mathbf{S} \delta
\end{aligned}
$$

where $\delta, \mathrm{T}$ are 3D variables of divergence, temperature, and $\mathrm{p}$ is surface pressure, and $\varphi$ is the corresponding mass variable. $\mathbf{H}, \mathbf{A}, \mathbf{B}$, and $\mathbf{S}$ are constant matrices, derived from the particular vertical discretization of the model. To obtain the vertical modes, we first obtain an equation only in $\delta$ by differentiating (B1) in time and substituting from (B3), (B4), using definition (B2), to get 


$$
\begin{gathered}
\frac{\partial^{2} \delta}{\partial t^{2}}=-\mathbf{Q} \nabla^{2} \delta \\
\mathbf{Q}=\mathbf{H S}+\mathbf{A B} \quad \text { (B6) }
\end{gathered}
$$

Let

$$
\mathbf{Q}=\mathbf{U E V}^{\mathrm{T}} \text { (B7) }
$$

be the eigen-decomposition of $\mathbf{Q}$, where $\mathbf{U}$ is the eigenvector matrix, $\mathbf{E}$ is the diagonal matrix of eigenvalues, and $\mathbf{V}$ is the matrix of eigenvectors of $\mathbf{Q}^{\mathrm{T}}\left(\mathbf{U V}^{\mathrm{T}}=\mathbf{I}\right)$. (Note: for general real nonsymmetric matrices, $\mathbf{U}, \mathbf{E}$, and $\mathbf{V}$ are complex in general, but for 3D hydrostatic models with sigma coordinate in the vertical, $\mathbf{U}, \mathbf{E}$, and $\mathbf{V}$ are always real and $\mathbf{E}$ is strictly positive, so that $\mathbf{E}^{-1}$ exists.)

Then

$$
\frac{\partial^{2} \delta}{\partial t^{2}}=-\mathbf{U E V}^{\mathrm{T}} \nabla^{2} \delta
$$

or

$$
\frac{\partial^{2} \hat{\delta}}{\partial t^{2}}=-\mathbf{E} \nabla^{2} \hat{\delta}
$$

where we have used

$$
\begin{gathered}
\delta=\mathbf{U} \hat{\delta} \quad(\mathrm{B} 10) \\
\hat{\delta}=\mathbf{V}^{\mathrm{T}} \boldsymbol{\delta} \quad(\mathrm{B} 11)
\end{gathered}
$$

and $\hat{\delta}$ is the vertical transform of $\delta$.

Now, we also have that

$$
\varphi=\mathbf{U} \hat{\varphi} \quad(\mathrm{B} 12)
$$

But then 


$$
\mathbf{H p}+\mathbf{A T}=\mathbf{U} \hat{\varphi}(\mathbf{B} 13)
$$

so it appears that we cannot recover $\mathrm{p}$ and $\mathrm{T}$ without some extra condition. The following argument, pointed out by Machenhauer shows that no extra condition is required for infinitesimal perturbations.

Combining (B3) and (B4) yields

$$
\frac{\partial \varphi}{\partial t}=-\mathbf{Q} \delta
$$

Solving for $\delta$ yields

$$
\delta=-\mathbf{Q}^{-1} \frac{\partial \varphi}{\partial t}
$$

( $\mathbf{Q}^{-1}$ exists, because the eigenvalues of $\mathbf{Q}$ are greater than zero)

Substituting (B15) back into (B3) and (B4) yields

$$
\begin{aligned}
& \frac{\partial \mathrm{T}}{\partial t}=\mathbf{B} \mathbf{Q}^{-1} \frac{\partial \varphi}{\partial t} \\
& \frac{\partial \mathrm{p}}{\partial t}=\mathbf{S} \mathbf{Q}^{-1} \frac{\partial \varphi}{\partial t}
\end{aligned}
$$

We can integrate out the time derivative, yielding unambiguous results for infinitesimal perturbations,

$$
\begin{aligned}
& \Delta \mathrm{T}=\mathrm{BQ}^{-1} \Delta \varphi \\
& \Delta \mathrm{p}=\mathrm{SQ}^{-1} \Delta \varphi
\end{aligned}
$$

In terms of vertical mode amplitudes, we have

$$
\begin{aligned}
& \Delta \mathrm{T}=\mathbf{B U E}^{-1} \Delta \hat{\varphi} \\
& \Delta \mathrm{p}=\mathbf{S U E}^{-1} \Delta \hat{\varphi} \quad(\mathrm{B} 21)
\end{aligned}
$$


This is the method used in TLNMC to convert vertical mode balance corrections to increments of $\mathrm{T}$ and $\mathrm{p}$.

It should be noted that other methods of resolving this (apparent) problem involve the minimization of a variational problem, where the definition of $\mathbf{M}$ is introduced using an EulerLagrange variable. 


\section{REFERENCES}

Baer, F. and J. Tribbia, 1977: On complete filtering of gravity waves through nonlinear initialization. Mon. Wea. Rev., 105, 1536-1539.

Bourke, W., and J. L. McGregor, 1983: A nonlinear vertical mode initialization scheme for a limited area prediction model. Mon. Wea. Rev., 111, 2285--2297.

Briere, S., 1982: Nonlinear normal mode initialization of a limited area model. Mon. Wea. Rev., $110,1166-1186$.

Courtier, P., and O. Talagrand, 1990: Variational assimilation of meteorological observations with the direct and adjoint shallow-water equations. Tellus, 42, 531-549.

Daley, R., 1978: Variational nonlinear normal mode initialization. Tellus, 30, 201-218. , 1991: Atmospheric Data Analysis. Cambridge University Press. 457 pp.

Derber, J. C., 1989: A variational continuous assimilation technique. Mon. Wea. Rev., 117, 24372446.

Errico, R.M., T. Rosmond, and J.S. Goerss, 1993: A comparison of forecast analysis and initialization increments in an operational data assimilation system. Mon. Wea. Rev., 121, 579-588.

Fillion, L., and C. Temperton, 1989: Variational implicit normal mode initialization. Mon. Wea. Rev., 117, 2219-2229.

, M. Tanguay, E. Lapalme, B. Denis, Z. Liu, N. Ek, M. Desgagne, V. Lee, S. Pellerin, 2007: Case dependent implicit normal mode balance operators. ECMWF Workshop on Flow-dependent aspects of data assimilation, Reading, United Kingdom, 125-142. 
Gauthier, P., and J.-N. Thépaut, 2001: Impact of the digital filter as a weak constraint in the preoperational 4DVAR assimilation system of the Météo-France. Mon. Wea. Rev., 129, 2089-2102.

Huang, X.-Y., and P. Lynch, 1993: Diabatic digital-filtering initialization: Application to the HIRLAM model. Mon. Wea. Rev., 121, 589-603.

Juang, H.-M., 2005: Discrete generalized hybrid vertical coordinates by a mass, energy and angular momentum conserving vertical finite-differencing scheme. NCEP Office Note 445, $33 \mathrm{pp}$. [Available online at http://www.emc.ncep.noaa.gov/officenotes/.]

Liang, L., B. Wang, J. Chan, Y. Duan, D. Wang, Z, Zeng, and L. Ma, 2007: Tropical cyclone forecasting with a model-constrained 3D-Var. I: Description. Quart. J. Roy. Meteor. Soc., 133, 147-153.

Machenhauer, B., 1977: On the dynamics of gravity oscillations in a shallow water model, with application to normal mode initialization. Contrib. Atmos. Phys., 50, 253-271.

Parrish, D. F., and J. C. Derber, 1992: The National Meteorological Center's spectral statisticalinterpolation system. Mon. Wea. Rev., 120, 1747-1763.

Temperton, C., 1989: Implicit normal mode initialization for spectral models. Mon. Wea. Rev., $117,436-451$.

Wee, T.-K., and Y.-H. Kuo, 2004: Impact of a digital filter as a weak constraint in MM5 4DVAR: An observing system simulation experiment. Mon. Wea. Rev., 132, 543-559.

Williamson, D. L. and R.E. Dickinson, 1972: Periodic Updating of Meteorological Variables. $J$. Atmos. Sci., 29, 190-193.

, R. Daley, and T. Schlatter, 1981: The balance between mass and wind fields resulting from multivariate optimal interpolation. Mon. Wea. Rev., 109, 2357-2376. 

, and R. Daley, 1983: A unified analysis--initialization technique. Mon. Wea. Rev., 111, 1517-1536.

Wu, W.-S., D. F. Parrish, and R. J. Purser, 2002: Three-dimensional variational analysis with spatially inhomogeneous covariances. Mon. Wea. Rev., 130, 2905-2916. 


\section{LIST OF FIGURES}

Figure 1. The structure of the first five vertical modes calculated for a global mean temperature and pressure profile derived from the 64 level background for an analysis valid at 1200 UTC 09 October 2007. The first index is associated with the solid line with next four in order from two through five with squares, diamonds, triangles and circles respectively.

Figure 2. Temperature increment (analysis minus background) at $500 \mathrm{hPa}$ (contour interval 0.05 $\mathrm{K}$ ) resulting from a single $500 \mathrm{hPa}$ temperature at $45^{\circ} \mathrm{N}, 90^{\circ} \mathrm{W}$ with a $1 \mathrm{~K}$ residual and observation error for a) CTL analysis and b) TLNMC analysis as well as c) the analysis difference TLNMC-CTL of temperature (interval $0.005 \mathrm{~K}$, negative values dashed, zero contour omitted) valid at 1200 UTC 09 October 2007.

Figure 3. Cross section taken at $90^{\circ} \mathrm{W}$ of the zonal wind increment (interval $0.1 \mathrm{~ms}^{-1}$, negative values dashed, zero contour omitted) resulting from a single $500 \mathrm{hPa}$ temperature at $45^{\circ} \mathrm{N}$, $90^{\circ} \mathrm{W}$ with a $1 \mathrm{~K}$ residual and observation error for a) CTL analysis and b) TLNMC analysis as well as c) the analysis difference TLNMC-CTL of zonal wind (interval $0.05 \mathrm{~ms}^{-1}$, negative values dashed, zero contour omitted) valid at 1200 UTC 09 October 2007.

Figure 4. Same as in Fig. 3 but for the ageostrophic component of the zonal wind. The interval for the analysis difference in c) has been increased to $0.1 \mathrm{~ms}^{-1}$.

Figure 5. Zonal wind increment at $500 \mathrm{hPa}$ (interval $1.5 \mathrm{~ms}^{-1}$, negative values dashed, zero contour omitted) resulting from the assimilation of all operationally available observations within the six-hour time window for a) CTL and b) TLNMC analyses valid at 1200 UTC 09 October 2007. 
Figure 6. Zonal wind at $500 \mathrm{hPa}$ for a) the background (interval $10 \mathrm{~ms}^{-1}$, negative values dashed, zero contour omitted) as well as b) the analysis difference TLNMC-CTL (interval $0.75 \mathrm{~ms}^{-1}$, negative values dashed, zero contour omitted) valid at 1200 UTC 09 October 2007.

Figure 7. Difference TLNMC-CTL in analyzed meridional wind tendency (interval $2.5 \times 10^{-4}$ $\mathrm{ms}^{-2}$, negative values dashed, zero contour omitted) valid at 1200 UTC 09 October 2007.

Figure 8. Zonal mean of the RMS of analyzed surface pressure tendency $\left(\mathrm{mb} \mathrm{s}^{-1}\right)$ valid at 1200 UTC 09 October 2007 for the background (grey solid), CTL analysis (black dashed) and TLNMC analysis (black solid).

Figure 9. Difference TLNMC-CTL in the zonal mean of the RMS of the pressure vertical velocity increment $\left(\mathrm{Pa} \mathrm{s}^{-1}\right)$ for analyses valid at 1200 UTC 09 October 2007.

Figure 10. The a) norm of the gradient and b) total cost function by iteration number for CTL (black) and TLNMC:(grey) analyses valid at 1200 UTC 09 October 2007.

Figure 11. The RMS of the fit of surface pressure observations $(\mathrm{mb})$ by cycle for 16 November 2006 through 14 January 2007 to each background (solid) and analysis (dashed) for the fullycycled CTL (black) and TLNMC (grey) experiments.

Figure 12. The average $500 \mathrm{hPa}$ geopotential height anomaly correlation scores by forecast day for the CTL (black) and TLNMC (grey) experiments verifying daily between 01 December 2006 though 14 January 2007 in the a) Northern Hemisphere and b) Southern Hemisphere. Forecasts for each experiment were initialized at 00 UTC and verified against their own analyses.

Figure 13. Time series of $500 \mathrm{hPa}$ geopotential height anomaly correlation scores for 5-day forecasts for the CTL (black) and TLNMC (grey) experiments verifying in the a) Northern 
Hemisphere and b) Southern Hemisphere. Forecasts for each experiment were initialized at 00 UTC and verified against their own analyses.

Figure 14. Verification of precipitation relative to observations over North America as a function of threshold amounts for 3-day forecasts (average of 60-84 hour forecasts) verifying between 01 December 2006 until 12 January 2007 for the CTL (black) and TLNMC (grey) experiments. Verification includes the a) equitable threat score and b) bias skill score. The grey numbers above panel b) indicate the number of cases that went into the verification of each threshold category.

Figure 15. Time series of RMS vector wind error $\left(\mathrm{ms}^{-1}\right)$ in the tropics for 3-day forecasts for the CTL (black) and TLNMC (grey) experiments at a) $250 \mathrm{mb}$ and b) $850 \mathrm{mb}$. Forecasts for each experiment were initialized at 00 UTC and verified against their own analyses. 


\section{TABLES}

Table 1. The corresponding gravity wave phase speeds for the first five vertical modes. The reference temperature and pressure profile was calculated as the global mean at each of the 64 model levels from the background for an analysis valid at 1200 UTC 09 October 2007.

\begin{tabular}{|l|l|}
\hline $\begin{array}{l}\text { Vertical Mode } \\
\text { Number }\end{array}$ & $\begin{array}{l}\text { Gravity Wave } \\
\text { Phase Speed }(\mathrm{m} / \mathrm{s})\end{array}$ \\
\hline 1 & 313.89 \\
\hline 2 & 232.91 \\
\hline 3 & 165.45 \\
\hline 4 & 120.07 \\
\hline 5 & 91.19 \\
\hline
\end{tabular}




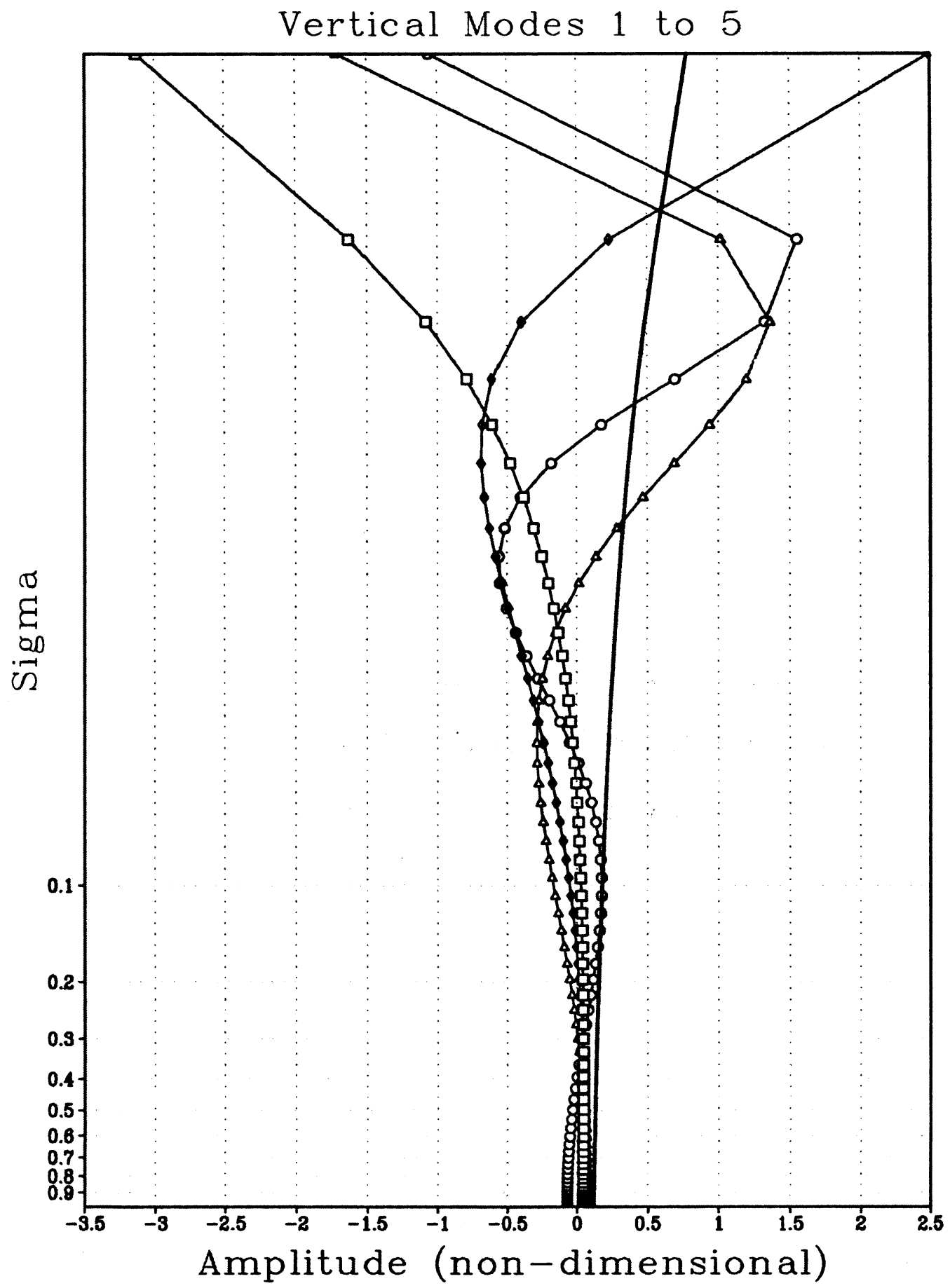

Figure 1. The structure of the first five vertical modes calculated for a global mean temperature and pressure profile derived from the 64 level background for an analysis valid at 1200 UTC 09 October 2007. The first index is associated with the solid line with next four in order from two through five with squares, diamonds, triangles and circles respectively. 

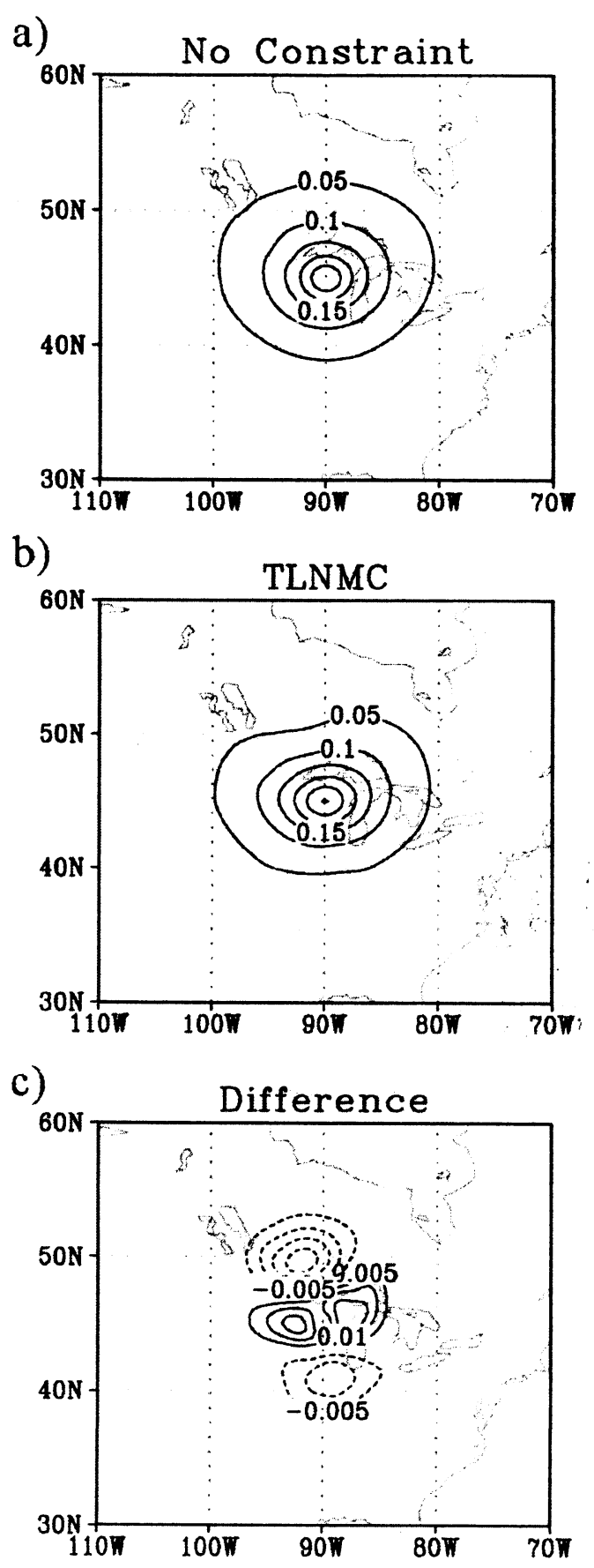

Figure 2. Temperature increment (analysis minus background) at $500 \mathrm{hPa}$ (contour interval $0.05 \mathrm{~K}$ ) resulting from a single $500 \mathrm{hPa}$ temperature at $45^{\circ} \mathrm{N}, 90^{\circ} \mathrm{W}$ with a $1 \mathrm{~K}$ residual and observation error for a) CTL analysis and b) TLNMC analysis as well as c) the analysis difference TLNMC-CTL of temperature (interval $0.005 \mathrm{~K}$, negative values dashed, zero contour omitted) valid at 1200 UTC 09 October 2007. 
a)

No Constraint

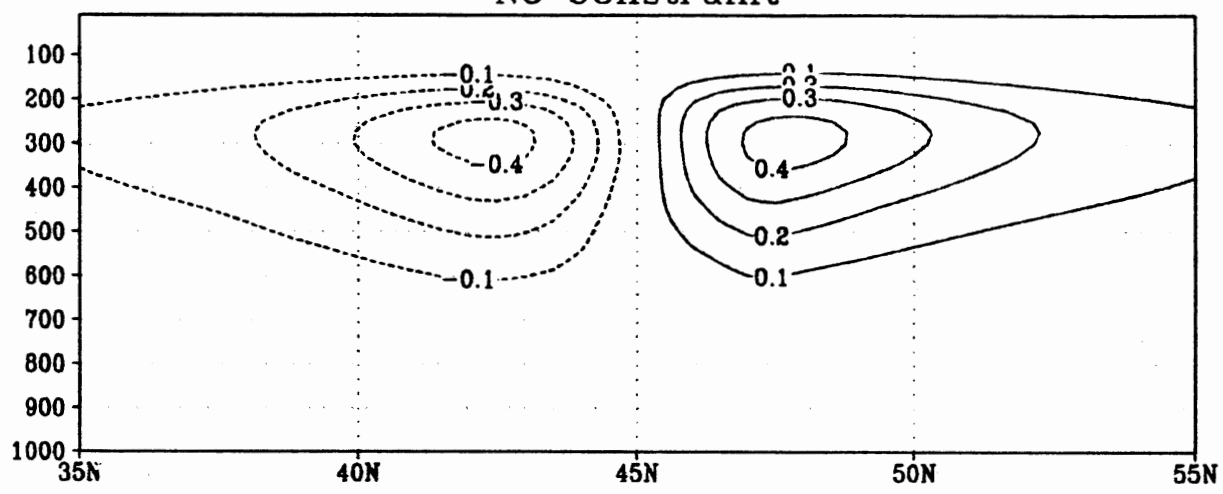

b)

TLNMC

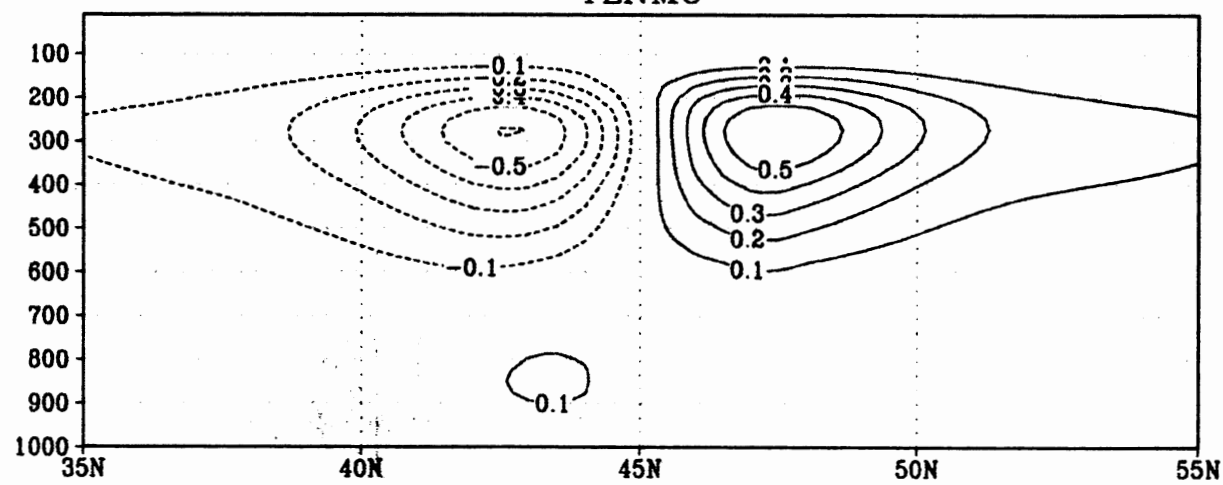

c)

Difference

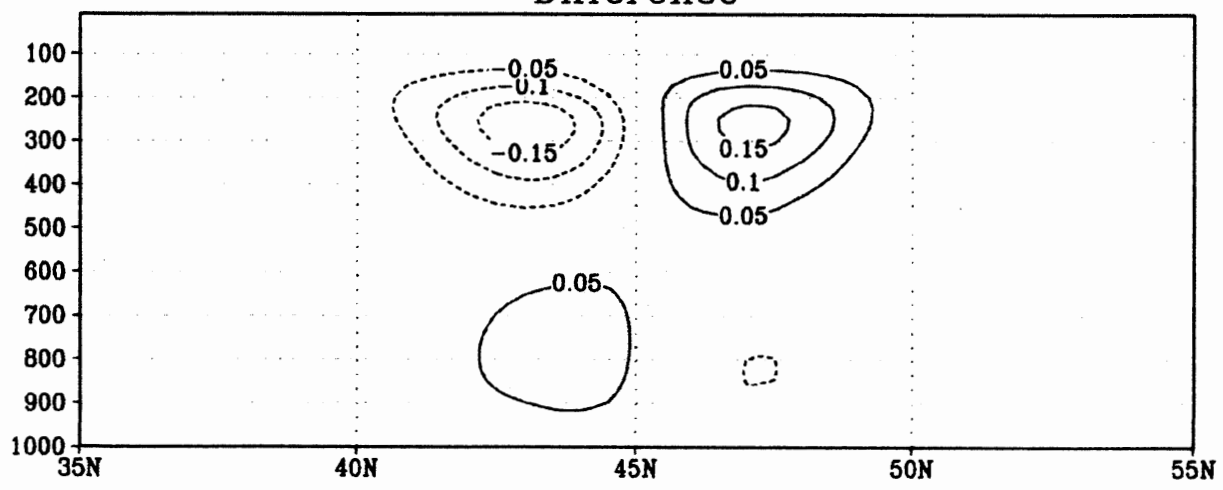

Figure 3. Cross section taken at $90^{\circ} \mathrm{W}$ of the zonal wind increment (interval $0.1 \mathrm{~ms}^{-1}$, negative values dashed, zero contour omitted) resulting from a single $500 \mathrm{hPa}$ temperature at $45^{\circ} \mathrm{N}, 90^{\circ} \mathrm{W}$ with a $1 \mathrm{~K}$ residual and observation error for a) CTL analysis and b) TLNMC analysis as well as c) the analysis difference TLNMC-CTL of zonal wind (interval $0.05 \mathrm{~ms}^{-1}$, negative values dashed, zero contour omitted) valid at 1200 UTC 09 October 2007. 


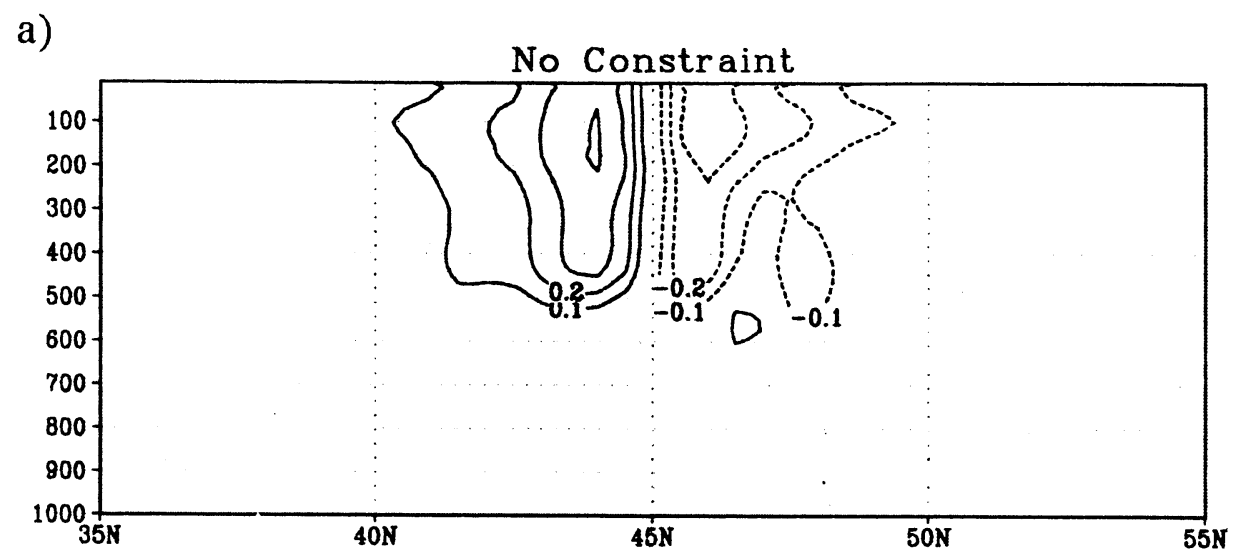

b)
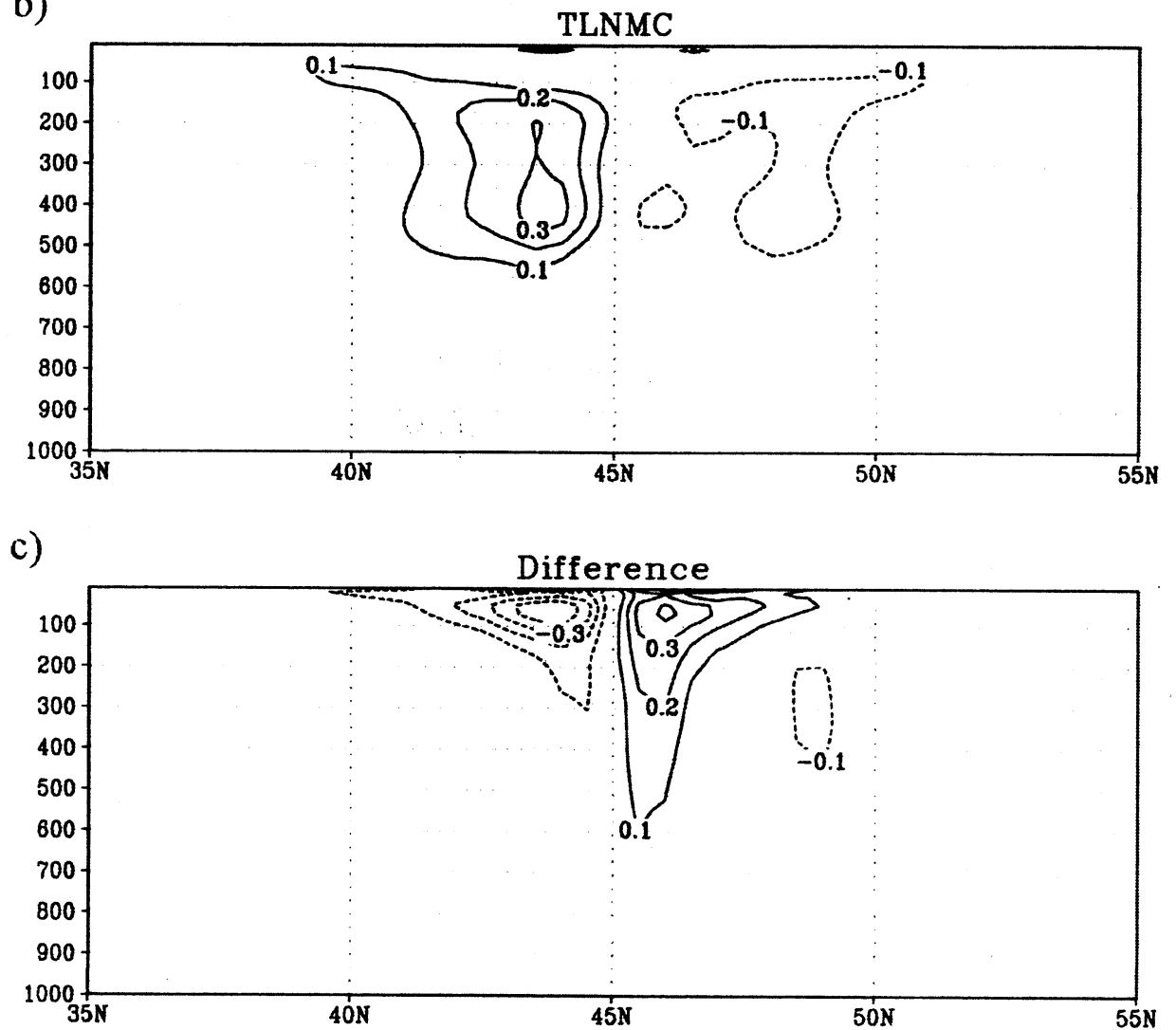

Figure 4. Same as in Fig. 3 but for the ageostrophic component of the zonal wind. The interval for the analysis difference in c) has been increased to $0.1 \mathrm{~ms}^{-1}$. 
a) No Constraint

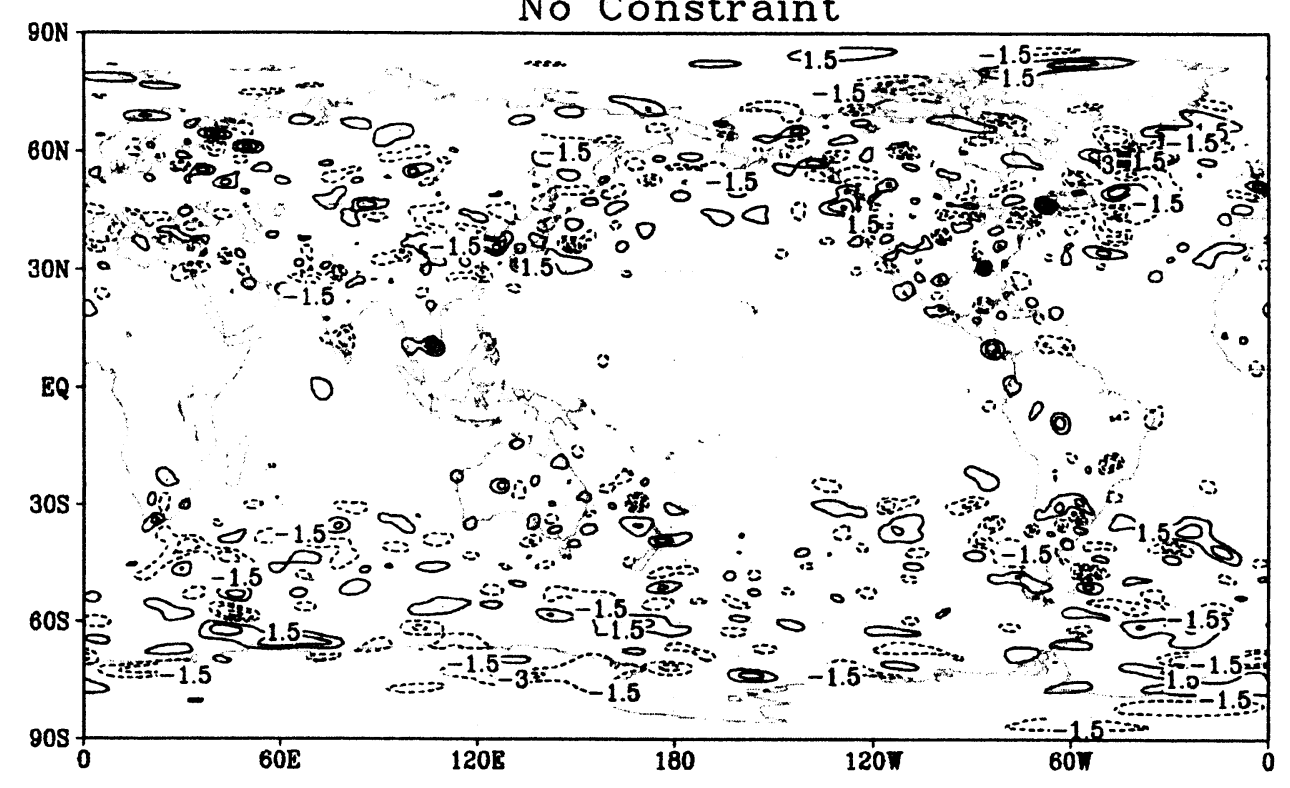

b)

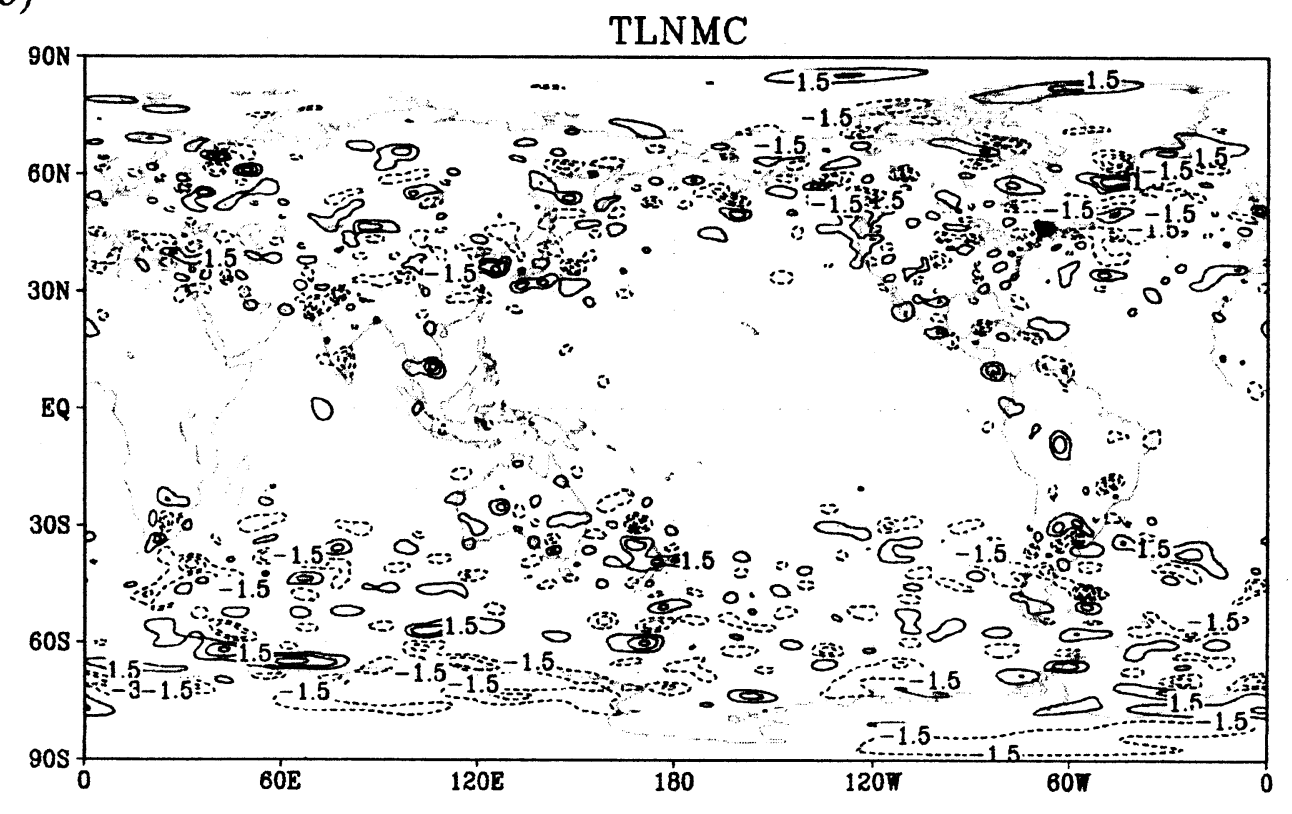

Figure 5. Zonal wind increment at $500 \mathrm{hPa}$ (interval $1.5 \mathrm{~ms}^{-1}$, negative values dashed, zero contour omitted) resulting from the assimilation of all operationally available observations within the six-hour time window for a) CTL and b) TLNMC analyses valid at 1200 UTC 09 October 2007. 
a)

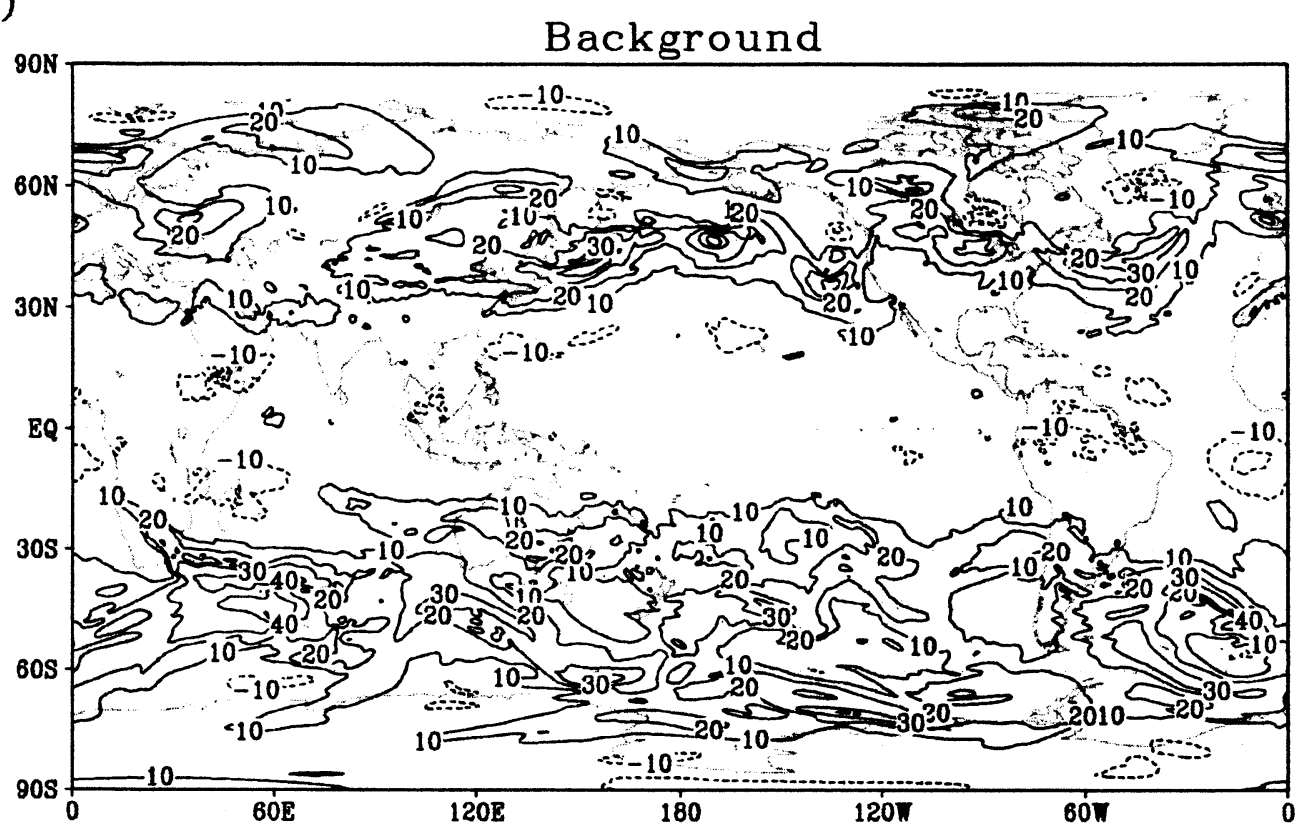

b)

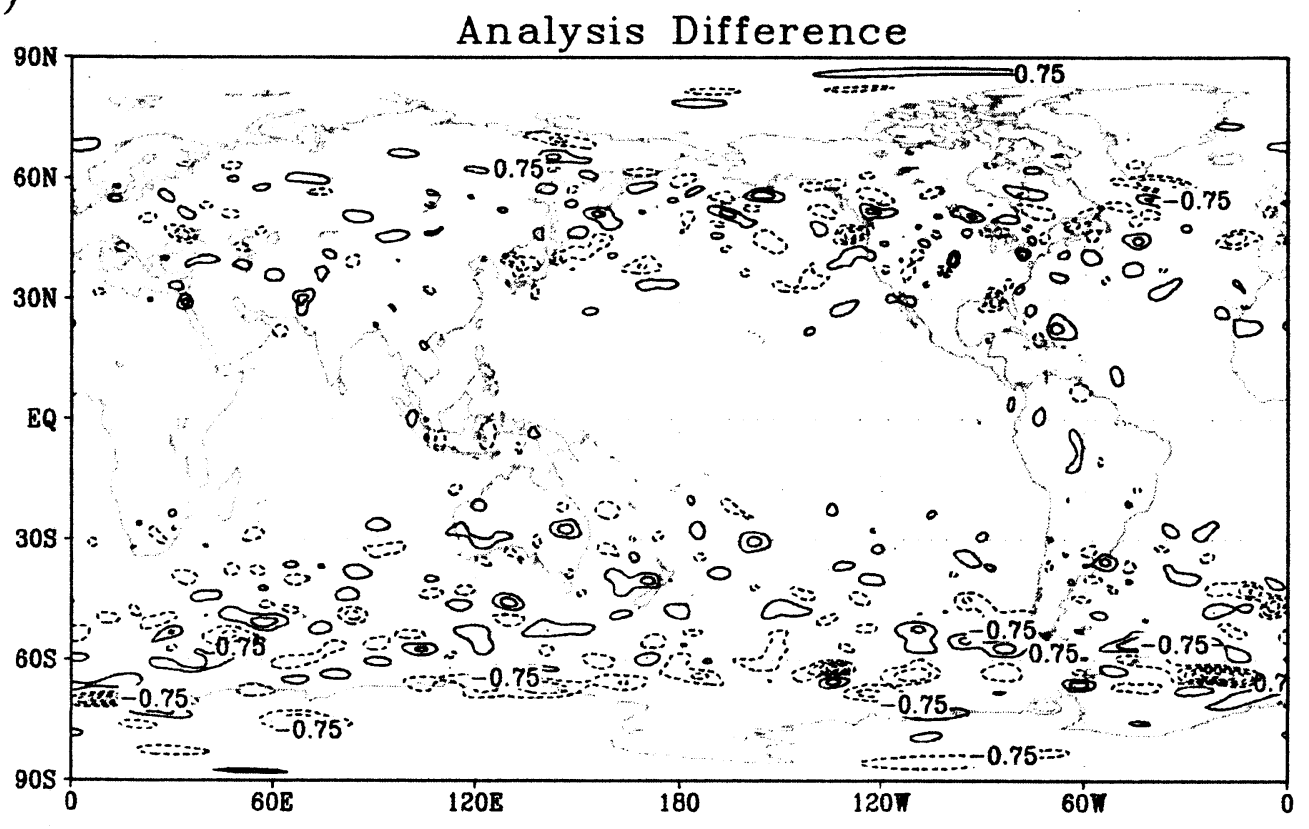

Figure 6. Zonal wind at $500 \mathrm{hPa}$ for a) the background (interval $10 \mathrm{~ms}^{-1}$, negative values dashed, zero contour omitted) as well as b) the analysis difference TLNMC-CTL (interval $0.75 \mathrm{~ms}^{-1}$, negative values dashed, zero contour omitted) valid at 1200 UTC 09 October 2007. 


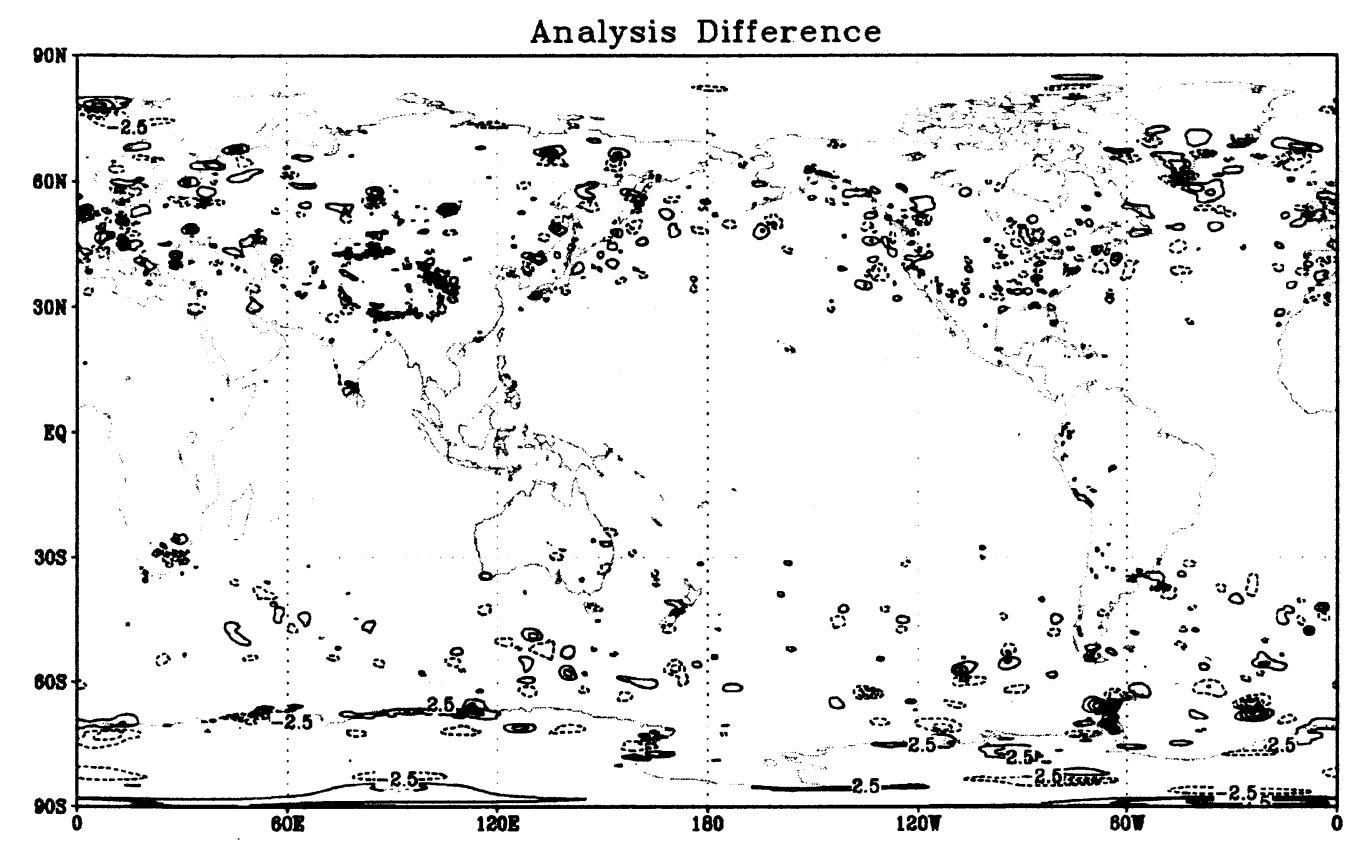

Figure 7. Difference TLNMC-CTL in analyzed meridional wind tendency (interval $2.5 \mathrm{x}$ $10^{-4} \mathrm{~ms}^{-2}$, negative values dashed, zero contour omitted) valid at 1200 UTC 09 October 2007. 


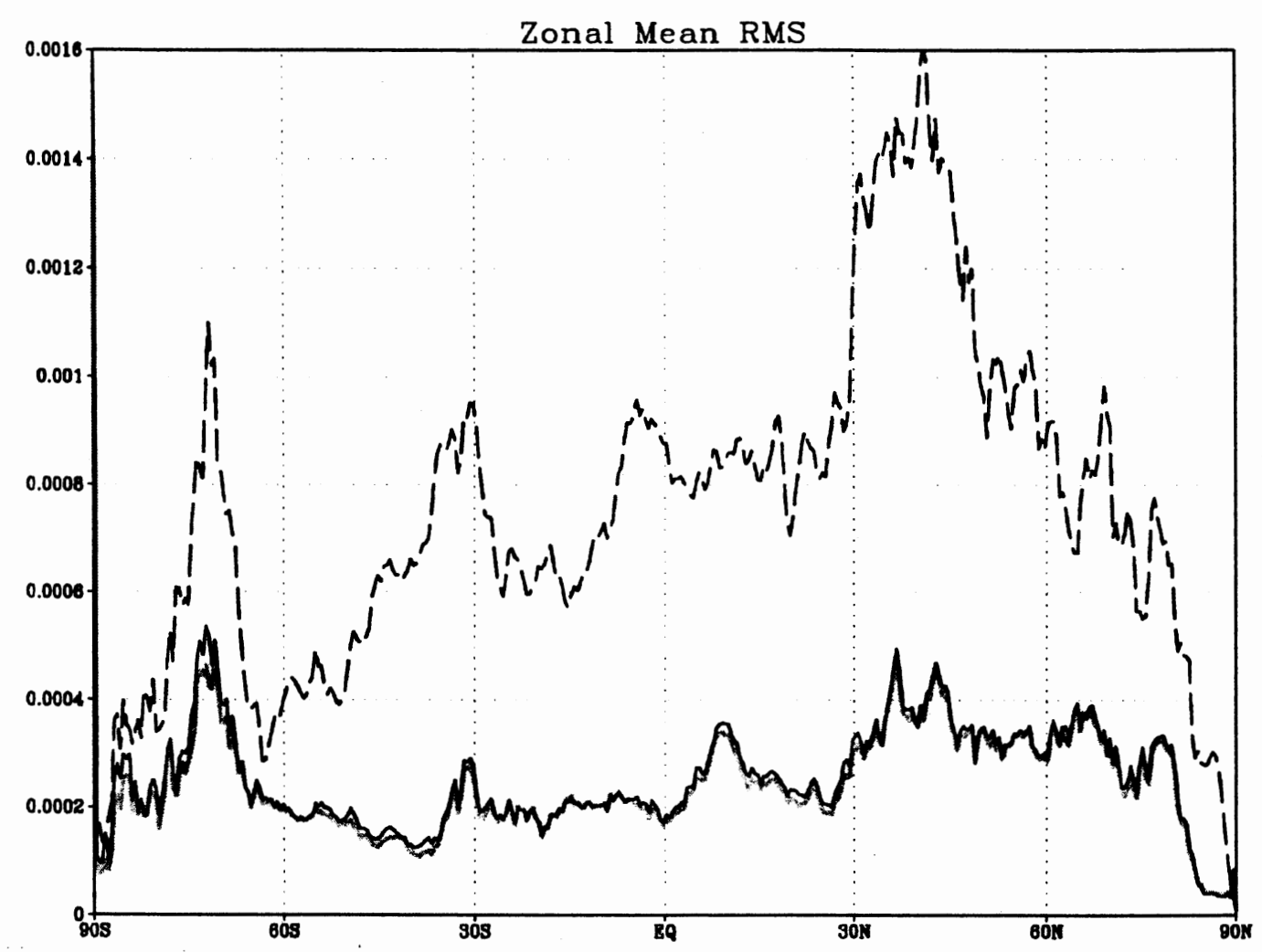

Figure 8. Zonal mean of the RMS of analyzed surface pressure tendency $\left(\mathrm{mb} \mathrm{s}^{-1}\right)$ valid at 1200 UTC 09 October 2007 for the background (grey solid), CTL analysis (black dashed) and TLNMC analysis (black solid). 


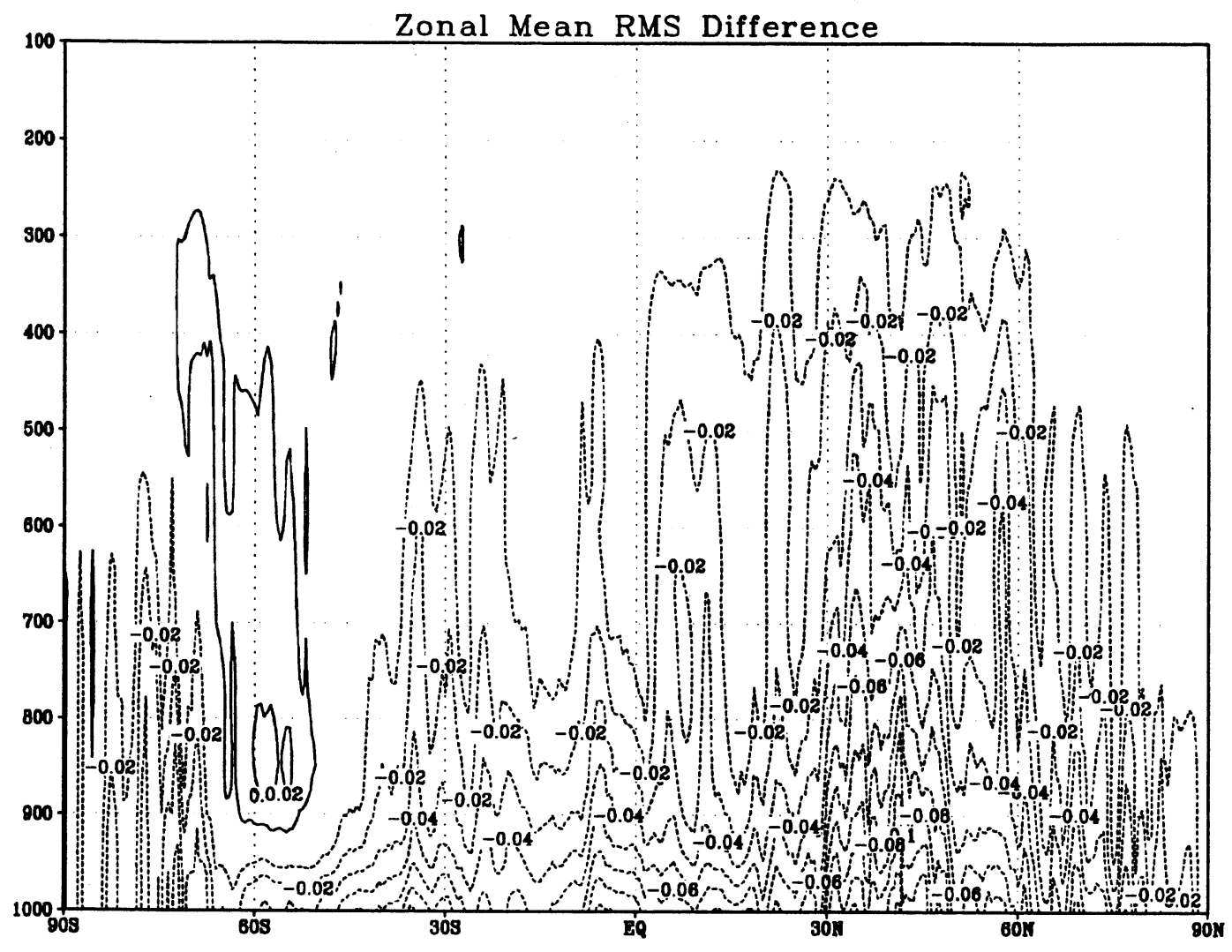

Figure 9. Difference TLNMC-CTL in the zonal mean of the RMS of the pressure vertical velocity increment $\left(\mathrm{Pa} \mathrm{s}^{-1}\right)$ for analyses valid at 1200 UTC 09 October 2007. 

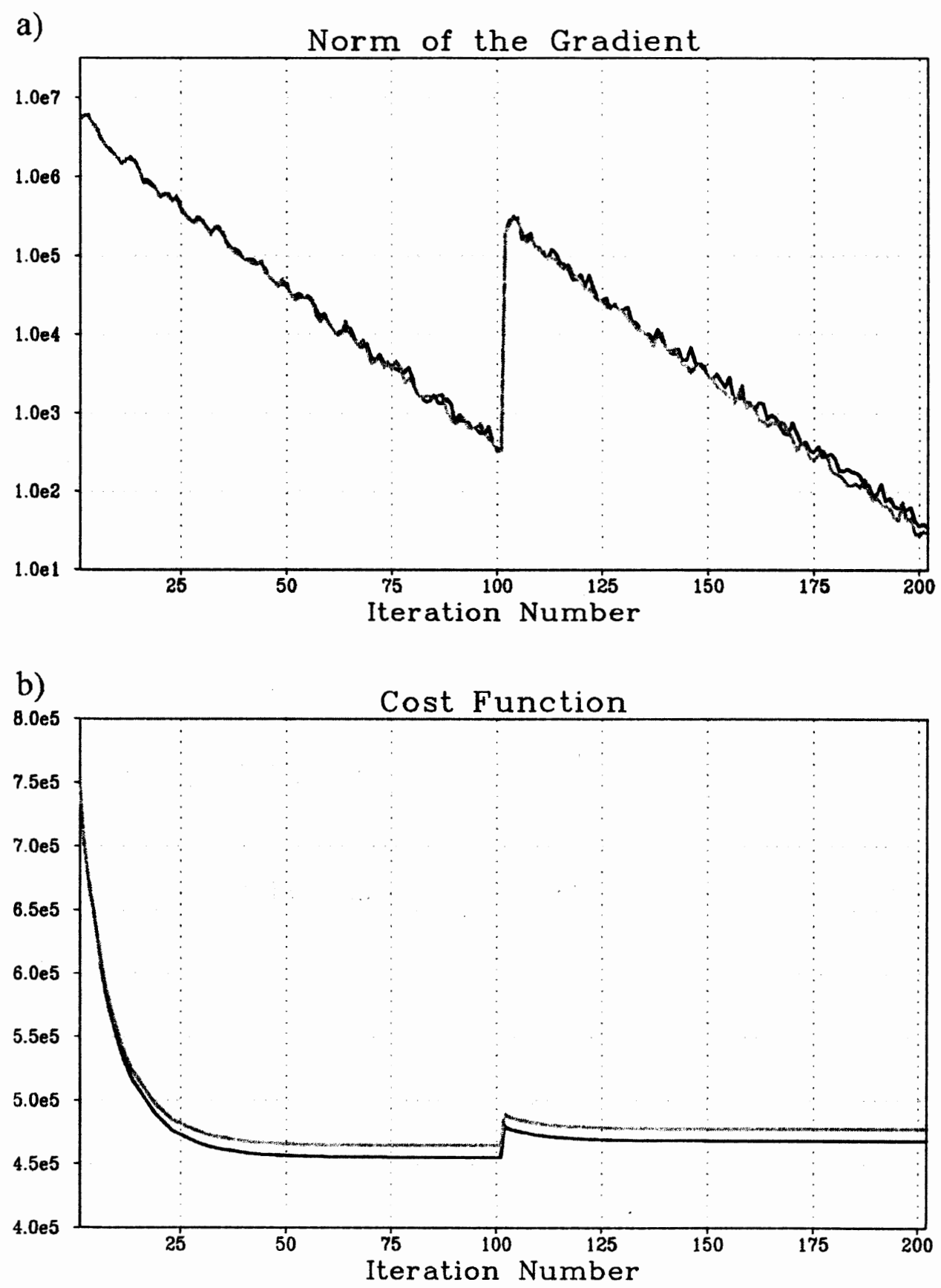

Figure 10. The a) norm of the gradient and b) total cost function by iteration number for CTL (black) and TLNMC (grey) analyses valid at 1200 UTC 09 October 2007. 


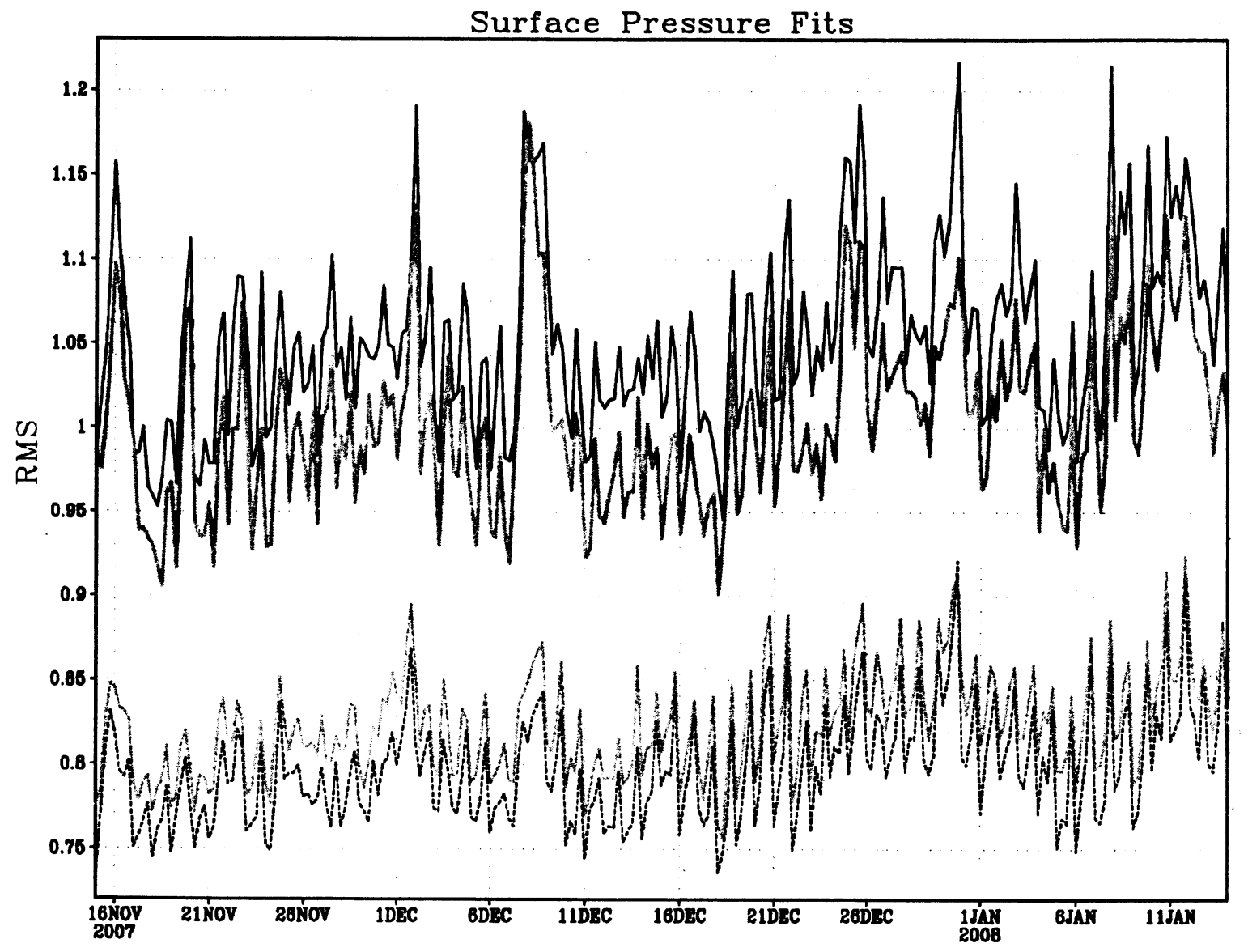

Figure 11. The RMS of the fit of surface pressure observations (mb) by cycle for 16 November 2006 through 14 January 2007 to each background (solid) and analysis (dashed) for the fully-cycled CTL (black) and TLNMC (grey) experiments. 

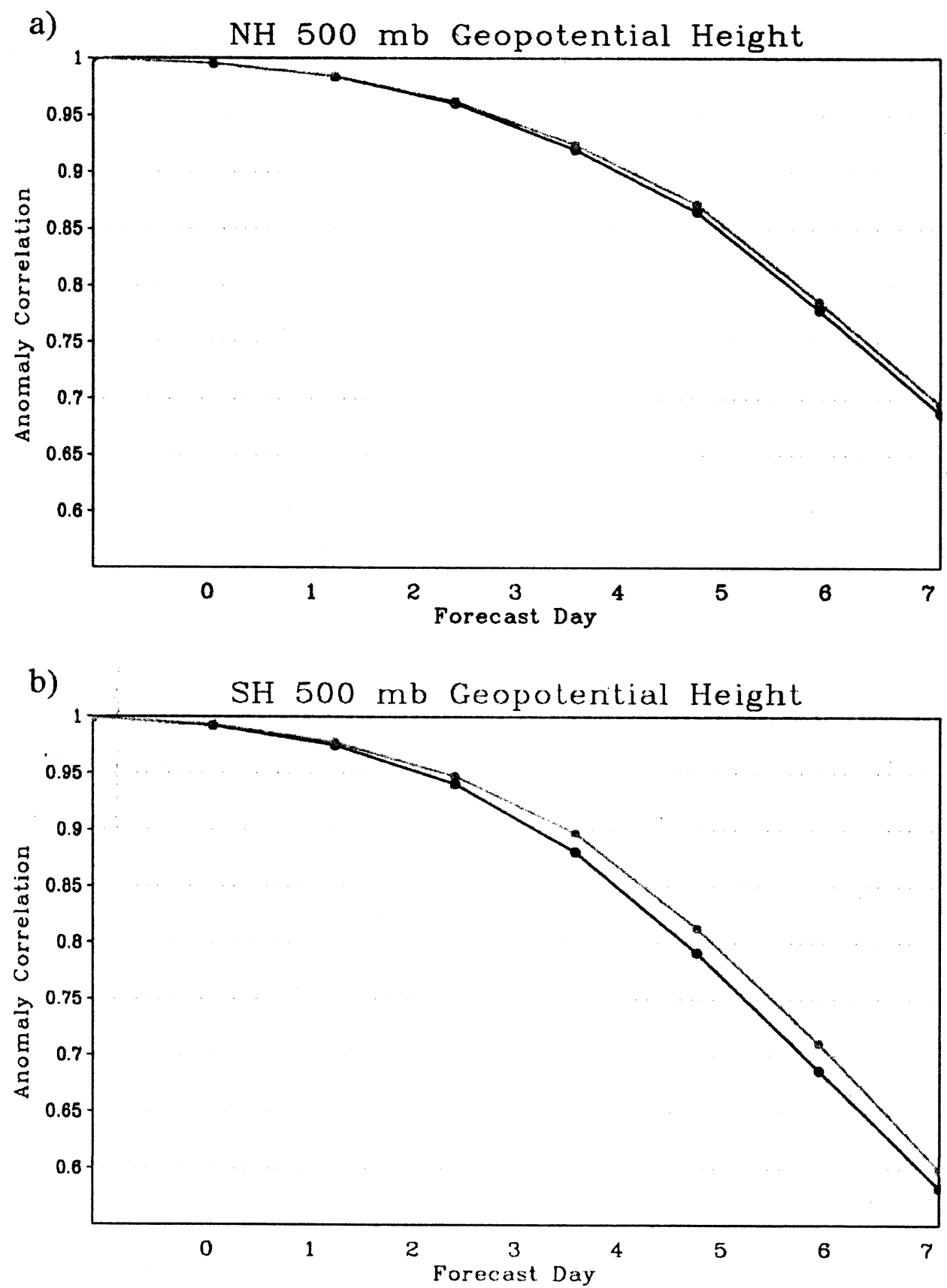

Figure 12. The average $500 \mathrm{hPa}$ geopotential height anomaly correlation scores by forecast day for the CTL (black) and TLNMC (grey) experiments verifying daily between 01 December 2006 though 14 January 2007 in the a) Northern Hemisphere and b) Southern Hemisphere. Forecasts for each experiment were initialized at 00 UTC and verified against their own analyses. 

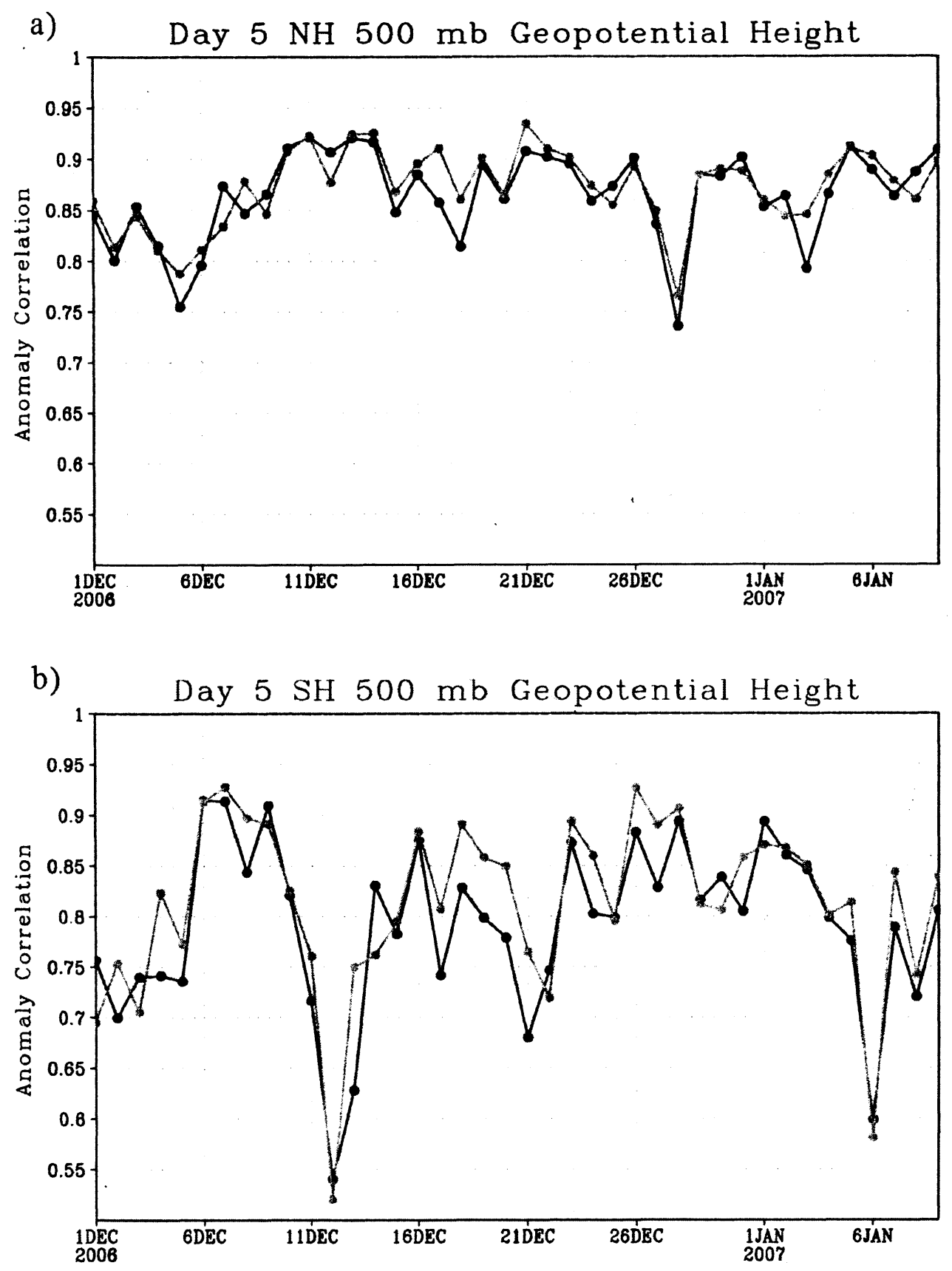

Figure 13. Time series of $500 \mathrm{hPa}$ geopotential height anomaly correlation scores for 5day forecasts for the CTL (black) and TLNMC (grey) experiments verifying in the a) Northern Hemisphere and b) Southern Hemisphere. Forecasts for each experiment were initialized at $00 \mathrm{UTC}$ and verified against their own analyses. 


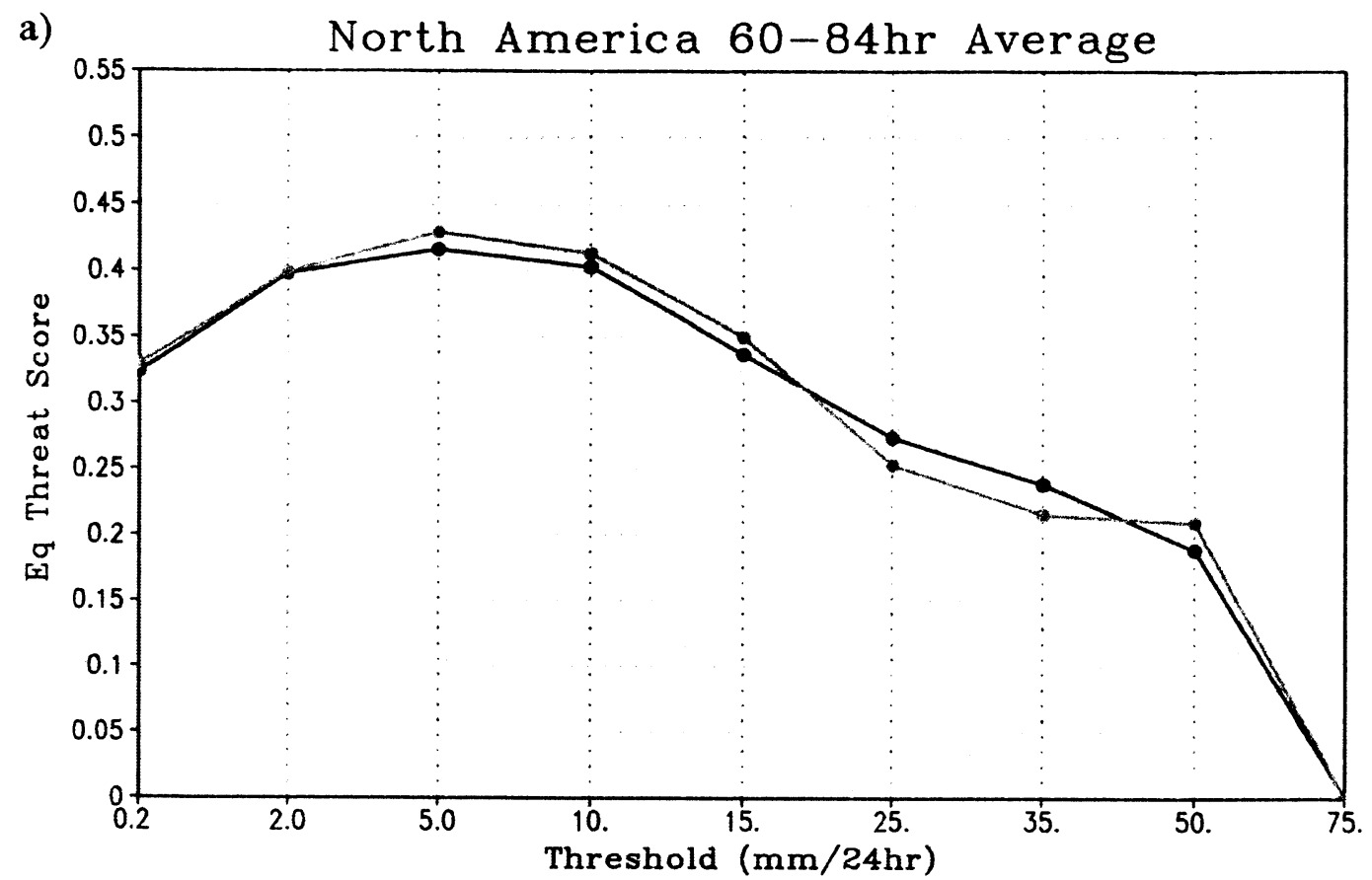

b)

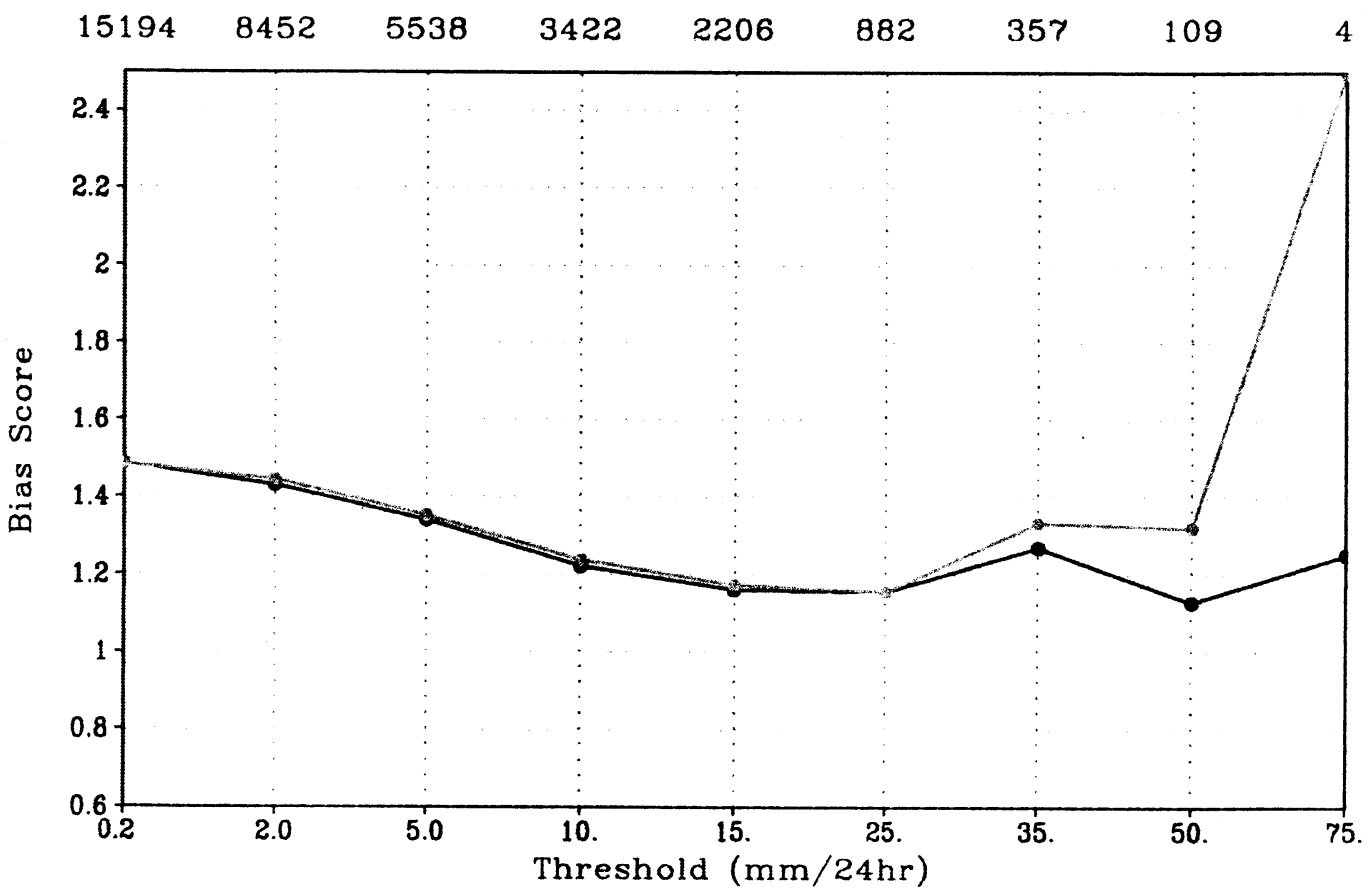

Figure 14. Verification of precipitation relative to observations over North America as a function of threshold amounts for 3-day forecasts (average of 60-84 hour forecasts) verifying between 01 December 2006 until 12 January 2007 for the CTL (black) and TLNMC (grey) experiments. Verification includes the a) equitable threat score and b) bias skill score. The grey numbers above panel b) indicate the number of cases that went into the verification of each threshold category. 


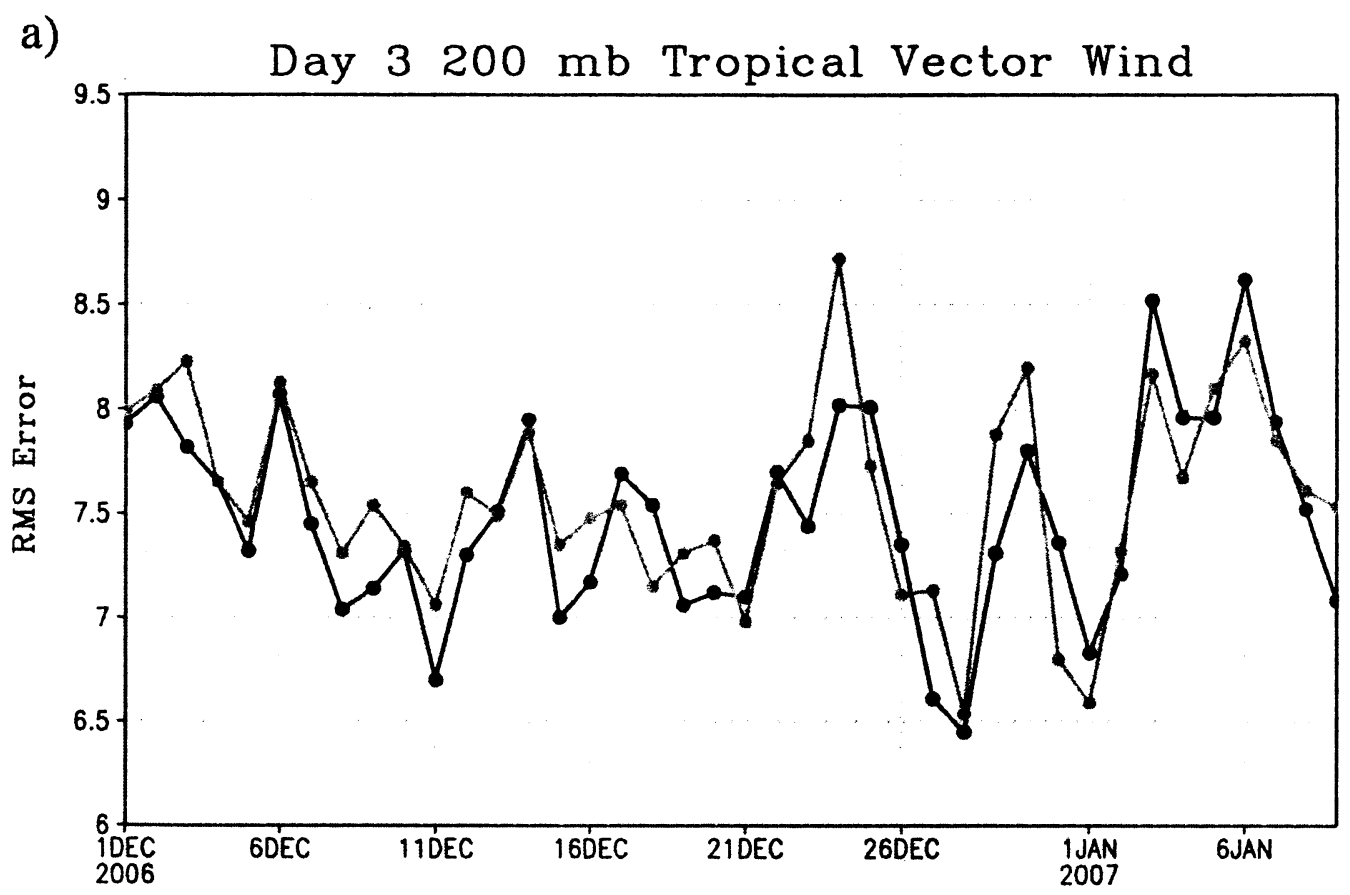

b)

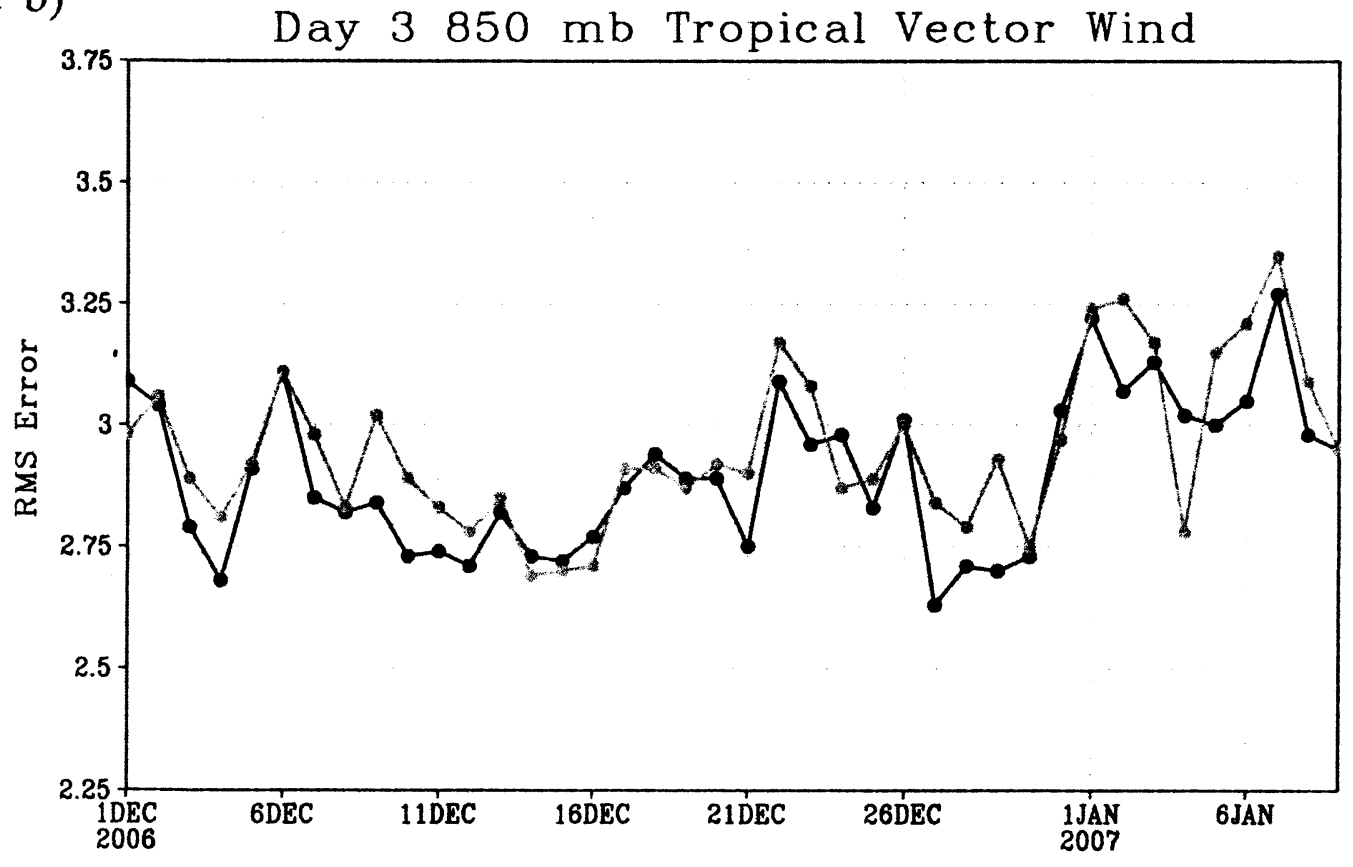

Figure 15. Time series of RMS vector wind error $\left(\mathrm{ms}^{-1}\right)$ in the tropics for 3-day forecasts for the CTL (black) and TLNMC (grey) experiments at a) $250 \mathrm{mb}$ and b) $850 \mathrm{mb}$. Forecasts for each experiment were initialized at 00 UTC and verified against their own analyses. 